\title{
On the evolution of the entropy and pressure profiles in X-ray luminous galaxy clusters at $z>0.4$
}

\author{
V. Ghirardini ${ }^{1,2}$, S. Ettori ${ }^{2,3}$, S. Amodeo ${ }^{4}$, R. Capasso ${ }^{5,6}$, and M. Sereno ${ }^{1,2}$ \\ 1 Dipartimento di Fisica e Astronomia Università di Bologna, via Piero Gobetti, 93/2, 40129 Bologna, Italy \\ e-mail: vittorio.ghirardini2@unibo.it \\ 2 INAF, Osservatorio Astronomico di Bologna, via Piero Gobetti, 93/3, 40129 Bologna, Italy \\ 3 INFN, Sezione di Bologna, viale Berti Pichat 6/2, 40127 Bologna, Italy \\ 4 LERMA, Observatoire de Paris, PSL Research University, CNRS, Sorbonne Universités, UPMC Univ. Paris 06, 75014 Paris, France \\ 5 Department of Physics, Ludwig-Maximilians-Universitaet, Scheinerstr. 1, 81679 Muenchen, Germany \\ 6 Excellence Cluster Universe, Boltzmannstr. 2, 85748 Garching, Germany
}

Received 7 December 2016 / Accepted 4 April 2017

\begin{abstract}
Context. Galaxy clusters are the most recent products of hierarchical accretion over cosmological scales. The gas accreted from the cosmic field is thermalized inside the cluster halo. Gas entropy and pressure are expected to have a self-similar behaviour with their radial distribution following a power law and a generalized Navarro-Frenk-White profile, respectively. This has also been shown in many different hydrodynamical simulations.

Aims. We derive the spatially resolved thermodynamical properties of $47 \mathrm{X}$-ray galaxy clusters observed with Chandra in the redshift range $0.4<z<1.2$, which is one of the largest samples investigated so far with X-ray spectroscopy and masses reconstructed via the hydrostatic equilibrium equation, with particular care taken to reconstruct the gas entropy and pressure radial profiles.

We search for deviation from the self-similar behaviour and look for possible evolution with redshift.

Methods. Under the assumption of a spherically symmetric distribution of the intracluster plasma, we combine the deprojected gas density and deprojected spectral temperature profiles via the hydrostatic equilibrium equation to constrain the concentration and scale radius, which are the parameters that describe a Navarro-Frenk-White profile for each of the clusters in our sample. The temperature profile, which combined with the observed gas density profile reproduces the best-fit mass model, is then used to reconstruct the profiles of entropy and pressure. These profiles cover a median radial interval of $\left[0.04 R_{500}-0.76 R_{500}\right]$. After interpolating on the same radial grid and partially extrapolating up to $R_{500}$, these profiles are then stacked in three independent redshift bins to increase the precision of the analysis. The gas mass fraction is then used to improve the self-similar behaviour of the profiles by reducing the scatter among the profiles by a factor 3 .

Results. The entropy and pressure profiles lie very close to the baseline prediction from gravitational structure formation. We show that these profiles deviate from the baseline prediction as function of redshift, in particular at $z>0.75$, where, in the central regions, we observe higher values of the entropy (by a factor of $\sim 2.2$ ) and systematically lower estimates (by a factor of $\sim 2.5$ ) of the pressure. The effective polytropic index, which retains information about the thermal distribution of the gas, shows a slight linear positive evolution with the redshift and concentration of the dark matter distribution. A prevalence of non-cool core, disturbed systems, as we observe at higher redshifts, can explain such behaviours.
\end{abstract}

Key words. galaxies: clusters: intracluster medium - galaxies: clusters: general - X-rays: galaxies: clusters - intergalactic medium

\section{Introduction}

Cosmic structures evolve hierarchically from primordial density fluctuations, growing to form larger and larger systems under the action of gravity. Clusters of galaxies are the biggest virialized structures in the Universe and aggregate as bound objects at a relatively late time $(z<3)$. The cosmic baryons fall into the gravitational potential of the cold dark matter (CDM) halo and under the action of the collapse and subsequent shocks, adiabatic compression, and turbulence heat up to the virial temperature of few $\mathrm{keV}$ and form a fully ionized X-ray emitting intracluster medium (ICM; Tozzi \& Norman 2001; Voit et al. 2005; Zhuravleva et al. 2014).

Modelling the ICM emission by thermal brehmsstrahlung allows observations in the X-ray band to provide a direct probe of the gas (electron) density, $n_{\mathrm{e}}$, and gas temperature, $T$. Assuming the perfect gas law and a monoatomic gas, the pressure is recovered as $P=T n_{\mathrm{e}}$ and the specific entropy as $K=T / n_{\mathrm{e}}^{2 / 3}$.

Entropy is a fundamental quantity to track the thermal history of a cluster, since it always rises when heat is produced (see Voit et al. 2005). In the presence of non-radiative processes only, low-entropy gas would sink to the centre of the cluster while high-entropy gas would expand, producing the typical self-similar radial distribution that follows a power law with a characteristic slope of 1.1 (e.g. Tozzi \& Norman 2001). Deviations from this predicted behaviour have been observed in the central region of clusters (see Ponman et al. 1999). Simulations show that non-gravitational cooling and heating processes, such as radiative cooling and subsequent AGN and supernovae feedback, break self-similarity in the inner region of galaxy clusters (e.g. Voit et al. 2005; McCarthy et al. 2017). To justify the observed deviations from self-similarity, Tozzi \& Norman (2001) 

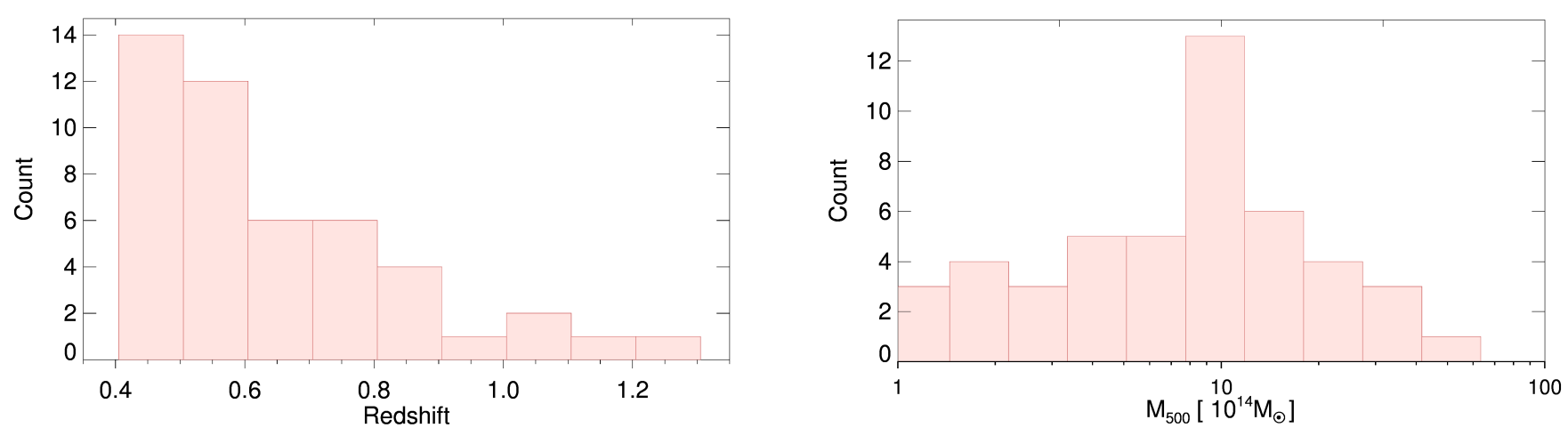

Fig. 1. Redshift (left) and total mass (right) distribution of the clusters in our sample.

propose a model in which an entropy floor is present before the gas is accreted by the dark matter halo.

Some dedicated papers have studied the evolution of the entropy profiles with redshift only in the last few years. McDonald et al. (2013), McDonald et al. (2014), and more recently Bartalucci et al. (2017) have concluded that both cool core clusters (CC) and non-cool core clusters (NCC) in samples selected through their Sunyaev-Zeldovich (Sunyaev \& Zeldovich 1980, hereafter SZ) signal have similar entropy profiles with no relevant changes with the cosmic time.

On the other hand, pressure is the quantity the least affected by the dynamical history and non-gravitational physics (Arnaud et al. 2010). Using the model introduced by Nagai et al. (2007), Arnaud et al. (2010) show that pressure has a very regular radial profile with only small deviations about the mean (less than 30 per cent outside the core regions). This is confirmed by recent observations of SZ-selected clusters (e.g. McDonald et al. 2014; Bartalucci et al. 2017) showing no significant deviation from the "universal profile" within $R_{500}$ up to redshift $z=0.6$.

In this work, we use the analysis of the mass distribution of 47 galaxy clusters in the redshift range $0.405-1.235$ presented in Amodeo et al. (2016) to study the radial shape of pressure and entropy at different redshifts, looking for deviations from the self-similar behaviour and for evolution with cosmic time.

In the present study, we assume a flat $\Lambda \mathrm{CDM}$ cosmology with matter density parameter $\Omega_{\mathrm{M}}=0.3$ and an Hubble constant of $H_{0}=70 \mathrm{~km} \mathrm{~s}^{-1} \mathrm{Mpc}^{-1}$.

Therefore the critical density of the Universe is written as

$\rho_{\mathrm{c}} \equiv \frac{3 H^{2}(z)}{8 \pi G}=\frac{3 H_{0}^{2}}{8 \pi G} E^{2}(z) \approx 136 \frac{M_{\odot}}{\mathrm{kpc}^{3}} E^{2}(z)$

where $E(z) \equiv H(z) / H_{0}=\left[\Omega_{\mathrm{M}}(1+z)^{3}+\Omega_{\Lambda}\right]^{1 / 2}$.

In our study, we also consider a rescaling dependent on the mass of the halo. To apply this, we measure the quantities of interest over the regions of the cluster defined by an overdensity $\Delta$, which is defined as a region for which the mean mass density is $\Delta$ times the critical density of the Universe.

In the following analysis, we choose $\Delta=500$, considering that our profiles have a radial extent of the same order of magnitude as $R_{500}$ (see Fig. C.3). By definition, $M_{500}$ is then equal to $4 / 3 \pi 500 \rho_{\mathrm{c}} R_{500}^{3}$.

All the quoted statistical uncertainties are at $1 \sigma$ level of confidence.

All the fitting processes are, unless otherwise stated, executed using the IDL routine MPFIT (Markwardt 2009), which performs a Levenberg-Marquardt least-squares fit weighting with both the errors on $x$ - and $y$-axes.
The paper is organized as follows: in Sect. 2 we present the sample, in Sect. 3 we present the method we apply to reconstruct the entropy and pressure profiles. The data analysis is detailed in Sect. 4 with an exhaustive discussion of our results presented in Sect. 5. We draw our conclusions in Sect. 6.

\section{Sample properties}

The sample selection and X-ray analysis is thoroughly explained in Amodeo et al. (2016). In this work, however, we recall some of the most important properties. Because they are massive objects, the relatively long Chandra exposure time ( $t_{\exp }>20 \mathrm{ks}$ ) considered for each cluster permits us to extract at least three independent spectra over the cluster's emission; this allows us to carry out a complete X-ray analysis as is usually performed for low redshift clusters. (see Amodeo et al. 2016, for details on the sample selection and X-ray analysis). We point out that clusters that are undergoing a major merger are excluded from the analysis since they would strongly break the hydrostatic equilibrium assumption, which is essential in recovering the mass profile. A completeness analysis of the sample has been made (see Amodeo et al. 2016): the selection criteria have effectively chosen the very massive high end of the cluster halo function in the investigated redshift range.

In Fig. 1, we show the redshift and mass distributions of the objects in our sample, with nine systems at $z>0.8$ and eight with an estimated $M_{500}$ larger than $2 \times 10^{15} M_{\odot}$. We use the centroid shift $w$ and the concentration $c_{\mathrm{SB}}$ (both resulting from the analysis made in Amodeo et al. 2016) to distinguish clusters with a cool core and clusters without it. We follow the results of Cassano et al. (2010), choosing clusters with $w<0.012$ and $c_{\mathrm{SB}}>0.2$ to be the classical relaxed CC. These objects are located in the upper-left quadrant of Fig. 2. The main goal of this paper is to study the evolution of entropy and pressure with cosmic time, therefore the sample is divided into redshift bins. Nevertheless a parallel analysis of the cluster sample has been made, in which clusters are divided in CC and NCC based on their morphological properties, using the criteria found by Cassano et al. (2010) mentioned above.

In our sample, the gas density profiles, obtained from the geometrical deprojection of the observed surface brightness profiles, cover the median radial range of $\left[0.04 R_{500}-0.76 R_{500}\right]$ with a mean relative error of $21 \%$. In Fig. C.3, we show the observed distribution of the minimum and maximum radius in the gas density profiles. We point out that half of the clusters have a radial coverage that extends above $0.77 R_{500}$. 


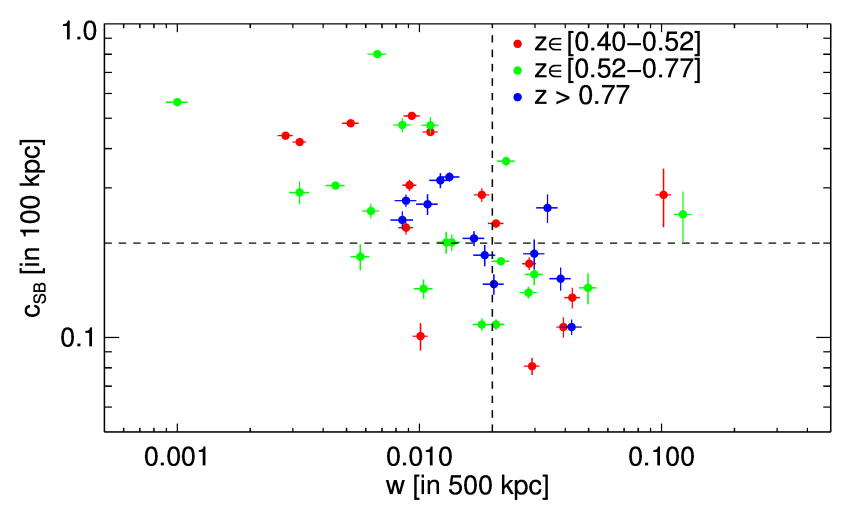

Fig. 2. Morphological parameters distribution in the plane of $w$ vs. concentration $c_{\mathrm{SB}}$.

\section{Method to reconstruct $K(r)$ and $P(r)$}

Amodeo et al. (2016) presented the method applied to constrain the mass distribution of the galaxy clusters in our sample under the assumption that a spherically symmetric ICM is in hydrostatic equilibrium with the underlying dark matter potential. The backward method adopted (see Ettori et al. 2013) allows us to constrain the parameters of a mass model, i.e. the concentration and scale radius for a NFW model (Navarro et al. 1997), using both the gas density profile, obtained from the geometrical deprojection of the X-ray surface brightness profile, and the spatially resolved spectroscopic measurements of the gas temperature. As a by-product of the best-fit mass model, we obtain the $3 \mathrm{D}$ temperature profile associated with the gas density measured in each radial bin. In other words, we obtain an estimate of the ICM temperature in each volume's shell where a gas density is measured from the geometrical deprojection of the X-ray surface brightness profile, in such a way that the best-fit mass model is reproduced by inserting the temperature and density profiles into the hydrostatic equilibrium equation (Binney \& Tremaine 1987). From the combination of these profiles, thermodynamical properties such as pressure and entropy are recovered.

Following Voit (2005), temperature, pressure, and entropy associated with this halo's overdensity are, respectively,

$$
\begin{aligned}
& k_{\mathrm{B}} T_{500}=10.3 \mathrm{keV}\left(\frac{M_{500}}{1.43 \times 10^{15} M_{\odot}}\right)^{\frac{2}{3}} E(z)^{2 / 3} \\
& P_{500}=1.65 \times 10^{-3} \mathrm{keV} \mathrm{cm}^{-3}\left(\frac{M_{500}}{3 \times 10^{14} M_{\odot}}\right)^{2 / 3} E(z)^{8 / 3} \\
& K_{500}=103.4 \mathrm{keV} \mathrm{cm}\left(\frac{M_{500}}{10^{14} M_{\odot}}\right)^{2 / 3} E(z)^{-2 / 3} f_{\mathrm{b}}^{-2 / 3} \\
& K(R) / K_{500}=1.42\left(\frac{R}{R_{500}}\right)^{1.1},
\end{aligned}
$$

where Eq. (5) is the Voit et al. (2005) prediction for which the radial dependence of $K$ is rescaled from $\Delta=200$ to 500 with the ratio $\frac{R_{500}}{R_{200}}=0.66$, as predicted from a NFW mass model with $c_{200}=4$, which is typical for massive systems.

The importance of using a proper rescaling, which includes the gas mass fraction to reach self-similarity in the entropy profiles, is pointed out in the work of Pratt et al. (2010). The gas fraction, $f_{\text {gas }}(<r)=M_{\text {gas }}(<r) / M_{\text {tot }}(<r)$, is here defined as the ratio between the gas mass obtained from the integration of the gas density over the cluster's volume and hydrostatic mass and is thus reconstructed directly from our data. We show the

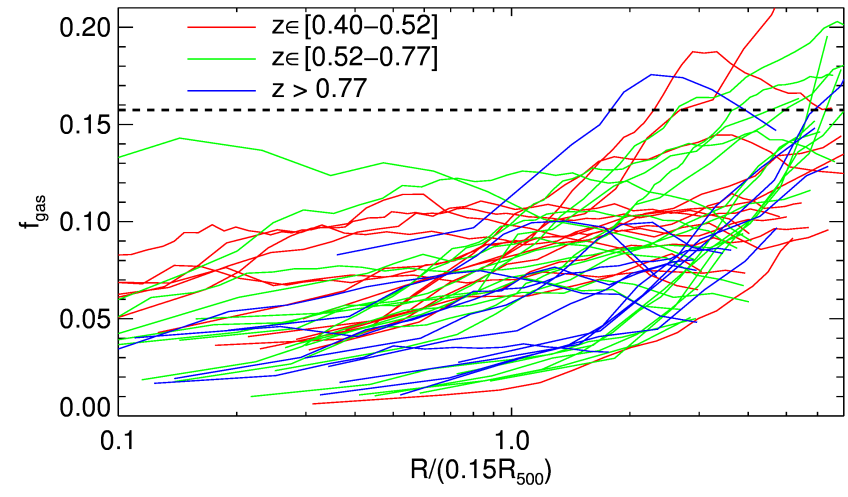

Fig. 3. Gas fraction profile for all our clusters. The black dashed line represents the universal baryon fraction from Planck (Planck Collaboration XIII 2016).

radial profiles of the gas fraction in Fig. 3. Throughout this paper we consider the universal baryon fraction to be $f_{\mathrm{b}}=0.15$ (Planck Collaboration XIII 2016). In Table A.1 we list additional information that is not presented in Amodeo et al. (2016), such as references on the redshifts and gas mass fraction at $R_{500}$.

\section{Data analysis}

We combine the profiles because of the poor statistics of each profile. In order to have at least one point in each radial bin, chosen logarithmically with total number of bins equal to $30^{1}$, over the range $\left[0.015 R_{500}, R_{500}\right]$, we first extrapolated the data using the best-fit power law plus a constant for the entropy profile and the functional form introduced by Nagai et al. (2007) for the pressure profile,

$\frac{P(x)}{P_{500}}=\frac{P_{0}}{\left(c_{500} x\right)^{\gamma}\left[1+\left(c_{500} x\right)^{\alpha}\right]^{\frac{\beta-\gamma}{\alpha}}}$,

where $x=R / R_{500}$ and $\gamma, \alpha$, and $\beta$ are the central slope, intermediate slope, and outer slope, respectively, defined by a scale parameter $r_{\mathrm{s}}=R_{500} / c_{500}\left(R \ll r_{\mathrm{s}}, R \sim r_{\mathrm{s}}\right.$ and $R \gg r_{\mathrm{s}}$, respectively). All the parameters of this function are left free except for the outer slope $\beta$ and the inner slope $\gamma$, which is fixed to the "universal" values (5.49 and 0.308; Arnaud et al. 2010).

We calculated the value of the thermodynamical quantities in the extrapolated radial points with the functional forms indicated above. The error associated with the thermodynamic quantities in the extrapolated radial points is the sum in quadrature of the propagated best-fitting parameters and the median uncertainty estimated in the last five radial bins of the raw profiles.

Furthermore, to investigate the average behaviour of these profiles as function of redshift, we divided the dataset into three redshift bins chosen to have approximatively the same number of clusters so that the resulting profiles have an approximatively constant signal-to-noise ratio: 15 with $z \in$ [0.4,0.52]; 20 with $z \in[0.52,0.77]$; and 12 with $z>0.77$. In each redshift bin, the profiles are stacked in logarithmic space using the inverse of the $1 \sigma$ error as weights, meaning that at each radial point the weighted mean is :

$\langle x\rangle=\frac{\Sigma w_{i} x_{i}}{\Sigma w_{i}}$ with $w_{i}=\sigma_{i}^{-2}$,

1 The number of bins equal to 30 is chosen to guarantee a proper radial coverage. 

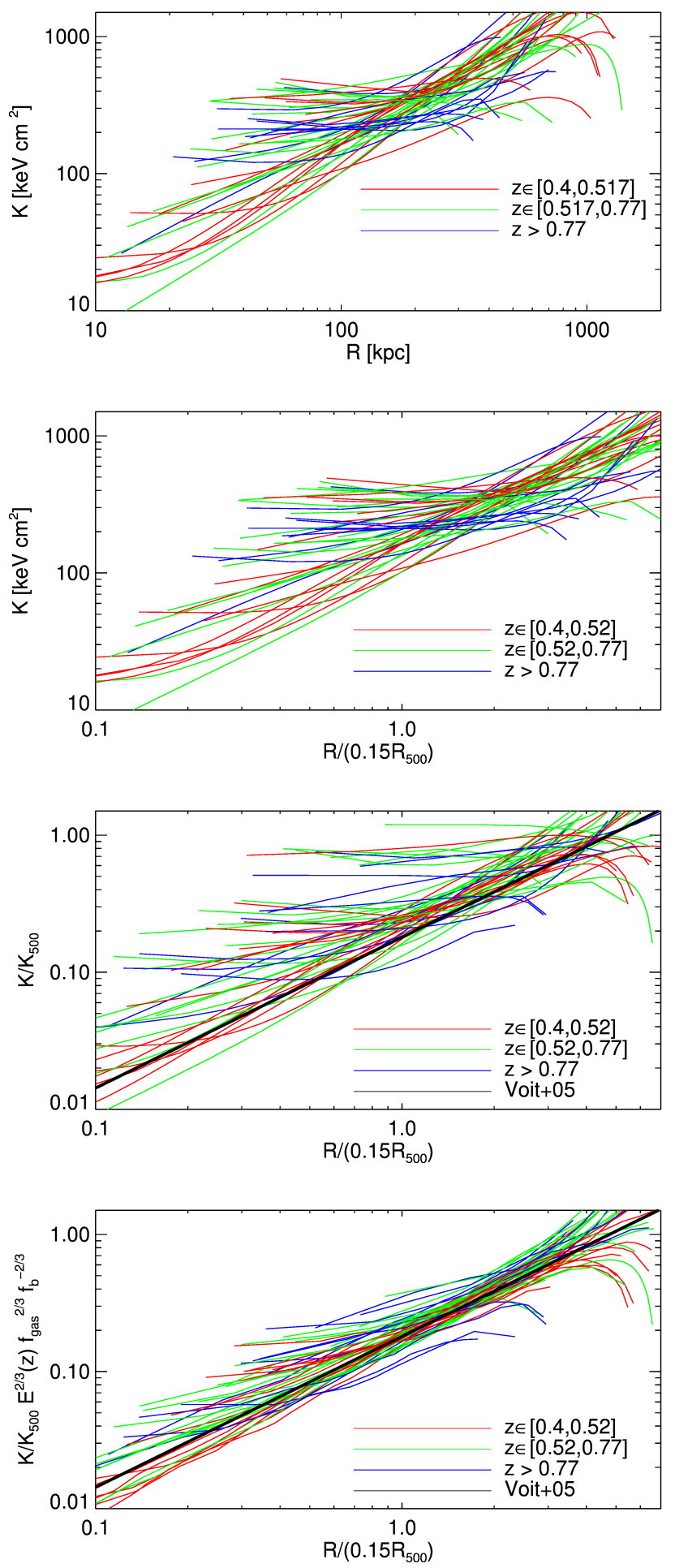

Fig. 4. Complete sample of our data is plotted and colour coded with respect to redshift. We can see the effect of rescaling from top to bottom, from largely scattered data to coherent data. No error bars are drawn for sake of clarity.

where $x$ is the logarithm of the considered thermodynamic quantities (pressure or entropy).

This "stacking" procedure ensures a higher statistical significance of the measured "mean" quantities, which propagates into a lower uncertainties in constraining the best-fit parameters of the functional forms adopted.

\subsection{Fitting procedure for the entropy profile}

In Fig. C.5, we show the reconstructed entropy profiles, rescaled as described below using $K_{500}$, of the 47 clusters in our sample prior to the application of the extrapolation and stacking procedure.

The radial behaviour of the entropy distribution is commonly described with a power law plus a constant term accounting for the combined action of cooling and heating feedbacks, which affect the central regions (see Cavagnolo et al. 2009),

$K=K_{0}+K_{100}\left(\frac{R}{100 \mathrm{kpc}}\right)^{\alpha}$.

This functional form has an underlaying physical sense when we rescale with $100 \mathrm{kpc}$, since typically deviations from nonradiative simulations are seen below this radius, where cool core clusters and non-cool core clusters actually differ. The parameter $K_{0}$ is the central entropy, which has been used in several works (i.e. Cavagnolo et al. 2009; Voit et al. 2005; McDonald et al. 2013) to discriminate between relaxed CC clusters, with $K_{0} \sim$ $30 \mathrm{keV} \mathrm{cm}{ }^{2}$, and disturbed NCC, with $K_{0}>70 \mathrm{keV} \mathrm{cm}^{2}$.

We also consider a functional form where the scaling is carried out with respect to $0.15 R_{500}$ to take into account the dimension of the core in systems at different mass and redshift as follows:

$\frac{K}{K_{500}}=K_{0}^{\prime}+K_{0.15}^{\prime}\left(\frac{R}{0.15 R_{500}}\right)^{\alpha^{\prime}}$.

As shown in Pratt et al. (2010), we expect the scatter among entropy profiles of the clusters to be suppressed even more when the renormalization includes both the global and radial dependence on the gas mass fraction,

$E(z)^{2 / 3} \frac{K}{K_{500}}\left(\frac{f_{\mathrm{gas}}}{f_{\mathrm{b}}}\right)^{2 / 3}=K_{0}^{\prime \prime}+K_{0.15}^{\prime \prime}\left(\frac{R}{0.15 R_{500}}\right)^{\alpha^{\prime \prime}}$.

In Fig. 4 we show how our refinement in the rescaling procedure of the entropy profiles from "no rescaling" at all to the inclusion of the dependence upon the gas fraction (see Eq. (9)), reduces the scatter among the profiles, improving the agreement with the self-similar prediction (Voit et al. 2005).

\subsection{No rescaling}

In the first panel of Fig. 4 we plot all the entropy profiles without applying any rescaling, i.e. the actual physical size in kpc versus entropy in $\mathrm{keV} \mathrm{cm}^{2}$. We name these profiles "raw data", because the physical values are reported without any rescaling. We can deduce that our clusters have very different thermodynamic histories and in fact at each radial point the profiles spans more than one order of magnitude, which excludes self-similarity.

We fit all the entropy profiles via Eq. (7) to look at the occupation of the parameter space in our sample. For each cluster we obtain a value for each one of the parameters $K_{0}, K_{100}$, and $\alpha$ and we plot all those values in Fig. C.1. We fitted each one of the three histograms with a lognormal distribution. We dedicated special attention to the parameter $K_{0}$, since the work of Cavagnolo et al. (2009) showed that central entropy may have a bimodal distribution. We obtained $\sim 10$ clusters with $K_{0}=0$ and $\sim 3$ with $\alpha=0$.

In the central regions of galaxy clusters, no significant emission from gas at very low temperature is observed (e.g. Peterson \& Fabian 2006), limiting the central value of the gas 


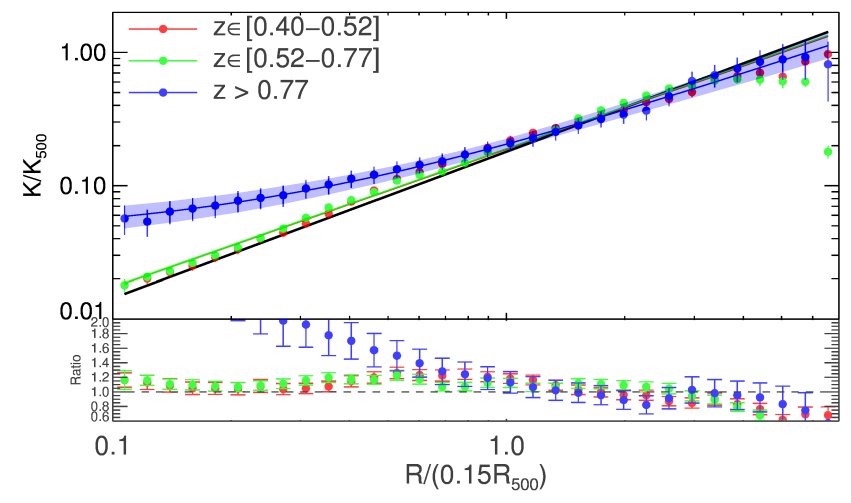

Fig. 5. Stacked entropy profiles and fits in the 3 redshift bins. The shaded areas represents the errors on the fit. The colours of the points are representative of the redshift bin considered. The black line represents the non-radiative prediction (Voit et al. 2005).

entropy to be positive $\left(K_{0}>0\right)$. For this reason these points are excluded from Fig. C.1.

The parameter $K_{0}$ (top panel) does not have a clear bimodal distribution. The data exhibit a peak between 150 and $200 \mathrm{keV} \mathrm{cm}^{2}$ and a significative tail for low values of $K_{0}$. The best fit obtained with two lognormal distributions shows one peak at $130 \mathrm{keV} \mathrm{cm}^{2}$ and one at $5 \mathrm{keV} \mathrm{cm}^{2}$, with $\chi_{\text {red }}^{2}=1.83$, while the unimodal fits yield a peak at $100 \mathrm{keV} \mathrm{cm}^{2}$ with $\chi_{\text {red }}^{2}=2.03$. We used the Bayesian information criterion (BIC; Schwarz 1978) to distinguish if there are statistical differences between the two fitted models: a $\triangle B I C$ between 2 and 6 indicates positive evidence against the model with higher BIC, while at values greater than 6 the evidence is strong. We obtained $\Delta \mathrm{BIC}=0.02$ and therefore we cannot discriminate between a unimodal and bimodal distribution. Even though the best-fit centers are close to the values found by Cavagnolo et al. (2009).

The distribution of the parameter $K_{100}$ (middle panel of Fig. C.1) is well fitted by a lognormal distribution with median value corresponding to $104 \pm 64{ }_{64}^{1702} \mathrm{keV} \mathrm{cm}{ }^{2}$ and $\chi_{\text {red }}^{2}=0.17$. The distribution of this parameter is smooth without any significative tail; this indicates that fitting considering $100 \mathrm{kpc}$ as the typical core dimension generates a well-defined distribution for $K_{100}$ resembling a lognormal distribution.

The distribution of the power law index $\alpha$ (bottom panel of Fig. C.1) is, as $K_{100}$, well fitted by a lognormal distribution with median value $1.40 \pm{ }_{0.45}^{0.672}$ and $\chi_{\text {red }}^{2}=1.1$. The visible peak has a higher value than the non-radiative prediction (Voit et al. 2005), but nevertheless it is compatible within $1 \sigma$.

\subsection{Rescaling using $K_{500}$}

The profile of a thermodynamical quantity, including entropy, should have a unique shape for galaxy clusters, after adequate rescaling (Voit 2005). Non-radiative simulations (see Voit et al. 2005) have shown that we should rescale with quantities defined with respect to the critical density in order to achieve this. As it is stated above, we use an overdensity of 500 and rescale the entropy profile using $K_{500}$, which is defined in Eq. (4). We can observe the effect of the scaling in the third panel of Fig. 4. The profiles we get are less scattered than the raw data, even though the dispersion about the mean is still quite high, about one order of magnitude. Nevertheless we observe that above $0.15 R_{500}$

\footnotetext{
2 This error represents the region that encompasses $68 \%$ of the data points from the best fit.
}

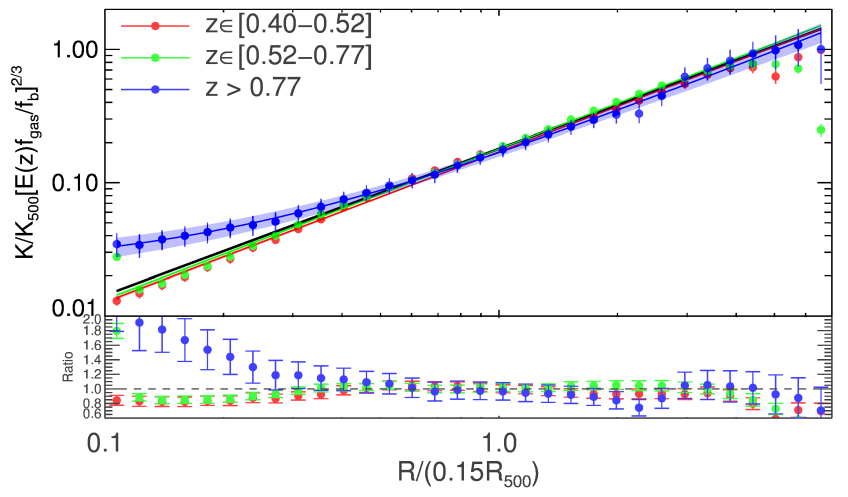

Fig. 6. Fits in different redshift bins showing the errors on the fits using the shaded area. The blue profile $(z>0.77)$ is compatible with the low redshift profiles for intermediate radii $\left(R>0.07 R_{500}\right)$ while at low radii there is a difference, where the high redshift entropy profile has a flat profile. The black line represents the non-radiative prediction (Voit et al. 2005).

most of the clusters have a self-similar behaviour. This is because non-gravitational processes are less relevant in the outskirts of galaxy clusters (Voit 2005).

As described in Sect. 4, we radially stack the data and we fit using Eq. (8). We show the regrouped data and the fitting results with their errors bars in Fig. 5 and in top part of Table 1. As we can observe from the ratio between the data and the predicted profile (bottom panel of Fig. 5), self-similarity is present below $0.6 R_{500}$ for the two low redshift bins while it is reached only between $0.15 R_{500}$ and $0.6 R_{500}$ at high redshift $(z>0.77)$. Moreover the high redshift stacked profile is slightly flatter than the others with a slope of $1.0 \pm 0.1$ and with its best fit requiring a constant term different from 0 to reproduce the data.

In the top part of Table 1 we show the best fit dividing the sample in CC and NCC. These parameters refers to Fig. B.1. As we can see both from the best fit and the plot, CC clusters are compatible with non-radiative prediction (Voit et al. 2005) with both slope and normalization. On the other hand, the NCC subset of clusters have a rather flat entropy profile.

\subsection{Rescaling using the gas fraction}

The entropy distribution depends on baryon fraction with a mass (or equivalently, temperature) dependence. Consequently entropy has both a radial and global dependence on the gas fraction (Pratt et al. 2010). When corrected by this effect, data become compatible with the non-radiative prediction (Voit et al. 2005) and the dispersion drops dramatically (last panel of Fig. 4).

A practical way to quantify the deviation from the selfsimilar prediction is shown in Fig. 7, where we show the behaviour of the dimensionless entropy profiles at some specific radii $\left(0.15 R_{500}, R_{2500} \text {, and } R_{1000}\right)^{2}$, by interpolating the surrounding data points, with respect to the mass. When the mass decreases the deviation from the self-similar prediction increases. By modelling this dependance with a simple power law, we obtain a slope value which becomes smaller (in modulus) at larger radii. In particular, we point out that at the highest radius considered $\left(R_{1000}\right)$, the profile is compatible with a constant, even though the influence of just 2 or 3 points makes the best-fit slope slightly negative.

2 Radii as big as $R_{500}$ are extrapolated and would not yield robust results and are therefore not shown. 


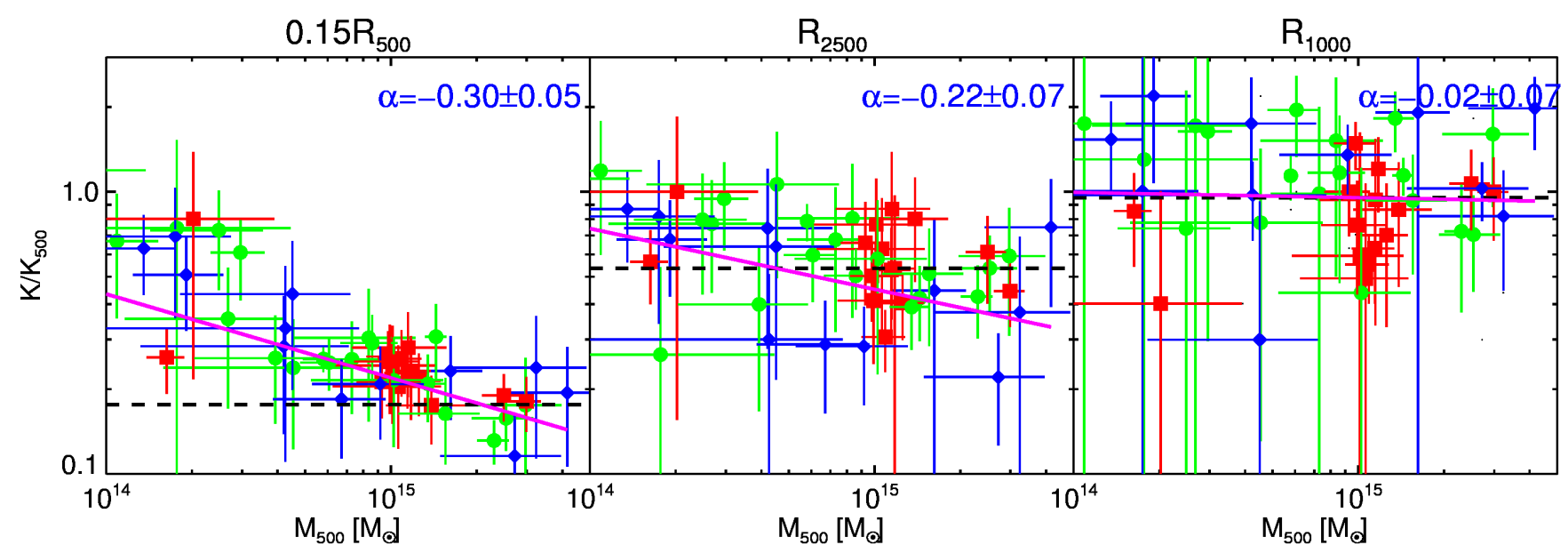

Fig. 7. Dimensionless entropy $K / K_{500}$ as a function of mass $M_{500}$ at different radii. Here the radii of $0.15 R_{500}, R_{2500}$, and $R_{1000}$ are used. The black dashed curve is the expectation from the self-similar model, and the magenta line is the best fit using a power law with index $\alpha$ on all the data points. Red, green, and blue points indicate data points from $z \in[0.4-0.52], z \in[0.52-0.77]$, and $z>0.77$ respectively.

We renormalized the entropy profiles multiplying them by gas fraction profiles $\left(K \rightarrow K \times\left(E(z) f_{\text {gas }}(R) / f_{\mathrm{b}}\right)^{2 / 3}\right)$. The resulting profiles are visible in the last panel of Fig. 4. The selfsimilarity of the entropy profile is now finally clear in our dataset of clusters at different redshift. At $0.15 R_{500}$, for instance, the scatter is reduced by a factor $\sim 3$ when the rescaling by the gas fraction is applied.

We then stack our profiles and we show the resulting entropy radial distribution in Fig. 6. We notice that the stacked profiles are compatible with the non-radiative prediction (Voit et al. $2005)$ in the radial range $\left[0.05 R_{500}-0.7 R_{500}\right]$, with the two low redshift stacked profile pushing this compatibility down to the lower limit of our analysis. Moreover at large radii, $R>0.7 R_{500}$, all the stacked profiles are slightly below the prediction.

We fit the stacked entropy profiles with a power law plus a constant and we show the results in Fig. 6 and in the bottom part of Table 1. The goodness of the fit has improved with respect to the rescaling without gas fraction and the parameters we get are closer to the non-radiative prediction.

The slope values we obtain are slightly larger than 1.1, indicating profiles that are steeper than the simulated profile, which agrees with several recent works (Voit et al. 2005; McDonald et al. 2013; Morandi \& Ettori 2007; Cavagnolo et al. 2009). The situation is different in the case of $z>0.77$, where the value of the central entropy is significantly different from zero. This indicates a high average central entropy for clusters at high redshifts.

In the bottom part of Table 1 we show the best fit dividing the sample into CC and NCC. This parameters refers to Fig. B.2, which is the plot analogous to Fig. 6. Similar to what happens to the split in redshift, the entropy profile of CC gets steeper than the prediction. The NCC are still flatter than the prediction and need a central entropy that is different from zero to fit the data.

\section{Discussion}

In the recent past, the dichotomy between cool core clusters, with a steep density profile and a drop of the temperature in the center, and non-cool core clusters, with a rather flat density profile and a flat temperature profile in the center, has been studied in numerous works. The particular shape of the density profile reflects in the behaviour of the entropy profile, where $\mathrm{CC}$ have a
Table 1. Best-fitting values and relative errors of the parameters of the models on entropy rescaled by $K_{500}$ (Top) (Eq. (8)) and on entropy rescaled by both $K_{500}$ and gas fraction (Bottom) (Eq. (9)).

\begin{tabular}{ccccc}
\hline \hline Subset & $K_{0.15}^{\prime}$ & $\alpha^{\prime}$ & $K_{0}^{\prime}$ & $\chi_{\text {red }}^{2}$ \\
\hline$z \in[0.4,0.52]$ & $0.188 \pm 0.003$ & $1.04 \pm 0.01$ & - & 2.34 \\
$z \in[0.52,0.77]$ & $0.189 \pm 0.002$ & $1.04 \pm 0.01$ & - & 2.0 \\
$z>0.77$ & $0.16 \pm 0.02$ & $1.0 \pm 0.1$ & $0.04 \pm 0.01$ & 0.14 \\
CC & $0.192 \pm 0.003$ & $1.12 \pm 0.02$ & - & 0.54 \\
NCC & $0.217 \pm 0.003$ & $0.77 \pm 0.01$ & - & 1.86 \\
\multicolumn{5}{c}{} \\
Subset & $K_{0.15}^{\prime \prime}$ & $\alpha^{\prime \prime}$ & $K_{0}^{\prime \prime}$ & $\chi_{\text {red }}^{2}$ \\
\hline$z \in[0.4,0.52]$ & $0.171 \pm 0.002$ & $1.13 \pm 0.01$ & - & 1.26 \\
$z \in[0.52,0.77]$ & $0.179 \pm 0.002$ & $1.13 \pm 0.01$ & - & 3.68 \\
$z>0.77$ & $0.15 \pm 0.01$ & $1.16 \pm 0.08$ & $0.022 \pm 0.005$ & 0.18 \\
CC & $0.173 \pm 0.002$ & $1.18 \pm 0.01$ & - & 0.46 \\
NCC & $0.184 \pm 0.005$ & $0.97 \pm 0.03$ & $0.006 \pm 0.003$ & 1.54 \\
\hline
\end{tabular}

Notes. In the bin $z>0.77$, we have a value for the central entropy that indicates the presence of many more NCC systems at high redshift. The exponent $\alpha$ and the term $K_{100}$ are compatible with the prediction of Voit et al. (2005). We also see that the goodness of the fit improves a lot when we correct by the gas fraction.

low entropy floor in the center while NCC have a higher entropy floor (see Figs. B.1 and B.2).

\subsection{Self-similarity}

Non-radiative simulations predict that the thermodynamic properties of clusters of galaxies should be self-similar once rescaled to specific physical quantities. In the previous section we have shown that by using a proper rescaling we reach self-similarity (see Fig. 4). Self-similarity is observed for all the redshift ranges. However, only a proper rescaling using the gas fraction makes the agreement with the prediction (Voit et al. 2005) within $20 \%$ above $0.05 R_{500}$. This is shown in the bottom panel of Figs. 5 and 6, where the ratios between data rescaled as indicated in Eqs. (8) and (9), respectively, and the non-radiative prediction (Voit et al. 2005) is shown. We observe that rescaling by the gas fraction is only needed in the high redshift bin to recover the self similar behaviour that is reached between 0.05 and $0.7 R_{500}$ within $20 \%$ from the theoretical value. 
The deviations present in the inner part of the profiles may be interpreted as some form of residual energy (Morandi \& Ettori 2007), which may be due to some non-gravitational physics processes.

Even though the agreement with simulations is remarkable, we get slightly steeper entropy profiles, i.e. $1.13 \pm 0.01$ for the low redshift bins, while we get a flatter profile in the high redshift bin, with a power law index that is compatible with the prediction, $1.16 \pm 0.08$, but with a non-zero central entropy.

The agreement with the results of Cavagnolo et al. (2009) is excellent in all three redshift bins since we find all slopes between 1.1 and 1.2. For the high redshift bins adding a constant to reproduce the inner part of the profile is required to make our results on the slope compatible with both non-radiative predictions (Voit et al. 2005) and observations (Cavagnolo et al. 2009). Otherwise a simple power law would yield a flat profile with slope $0.90 \pm 0.03$.

The excess with respect to the Voit et al. (2005) self-similar prediction is present at low radii where most of our data are above the prediction. This extra entropy is more pronounced in low mass systems, as shown in Fig. 7 and consistent with the results obtained by Pratt et al. (2010).

\subsection{Angular resolution effect}

In a thermalized system, low entropy gas sinks in the center while high entropy gas floats out in the outskirts, producing an entropy profile that increases monotonously with the radius. The net effect is that the larger the central bin considered for the analysis, the higher the measured value of the entropy. In the top panel of Fig. 8, we plot the value of $K_{0}$ for each cluster versus the radius of the innermost data point and measure an evident positive correlation (Pearson's rank correlation of 0.76 , corresponding to a significance of $8.6 \times 10^{-8}$ of its deviation from zero that is associated with the case with no correlation). This correlation becomes even more significative if we consider the same points rescaled by the properties of the halos. This is shown in the bottom panel of Fig. 8, where we measure a very significant Pearson's rank correlation of 0.83 . A similar result is shown by Panagoulia et al. (2014): the smaller the innermost radial bin, the smaller the central entropy we measure.

However, as we show in Fig. 8, the correlation between the rescaled central entropy and the innermost radial bin shows a slope value of $1.78 \pm 0.04$, which is about $8 \sigma$ away from the expected value, and therefore does not reproduce the predicted radial dependence from Voit et al. (2005; see Eq. (5)), suggesting that the flattening has a different origin form the lack of spatial resolution.

\subsection{Sample completeness}

In Amodeo et al. (2016), an extended study on the completeness of the sample analysed here is performed. In their Fig. 13, the completeness functions are compared to the distribution of the objects in the $M_{200}-z$ plane, showing that the applied selection criteria effectively selected the very massive high end of the cluster halo function in the investigated redshift range.

The observed trend to detect lower masses at higher redshifts is intrinsic to the halo mass function predicted for the hierarchical structure formation in the CDM dominated Universe.

Therefore, we conclude that, within the limits of our sample selection and statistics, the observed redshift and mass dependences are a reasonable representation of the behaviour of the
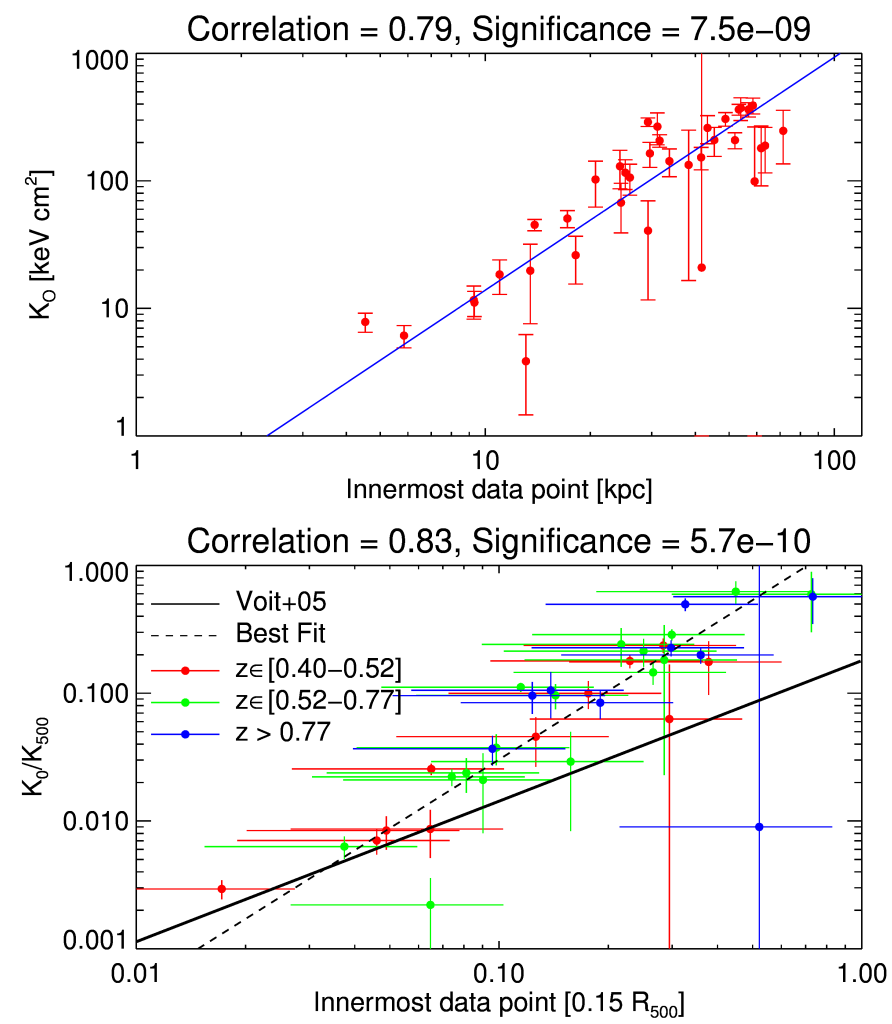

Fig. 8. Top: central entropy vs. the innermost data point. A clear postiive correlation is measured. Bottom: central entropy versus innermost data point rescaled, an even tighter correlation is present.

$\mathrm{X}$-ray luminous cluster population in the high mass end between $z=0.4$ and $z=1.2$.

\subsection{Bimodality}

Cavagnolo et al. (2009) have shown that the distribution of the values of the cluster central entropy reflects the dichotomy between CC and NCC clusters, finding two distinct populations peaking at $15 \mathrm{keV} \mathrm{cm}^{2}$ and $150 \mathrm{keV} \mathrm{cm}$. This bimodality has not been confirmed in later work (e.g. Santos et al. 2010; Pratt et al. 2010).

In Sect. 3.2 we have shown that using the BIC there are no statistical differences between a unimodal and bimodal distribution. We consider whether this lack of statistical evidence is because of the poor statistics of the cluster sample. We build a bootstrap analysis of our distribution by selecting 10000 samples of our objects (also allowing for repetitions). This approach permits us to determine whether a random sample extracted from our data show an evident bimodal distribution. The results of this analysis are shown in Fig. C.4. We observe a median value of $2.0_{-5.6}^{+3.2}$ and conclude that the distribution of this BIC is compatible with no differences between a unimodal and bimodal distribution.

In Fig. C. 2 the effect of redshift on the distribution of the central entropy is shown. As the number of clusters in each redshift bin is too small, we are not able to accurately prove any redshift evolution of the central entropy, however we have indications that an evolution may be present.

At high redshift we have an important peak at entropy higher than $100 \mathrm{keV} \mathrm{cm}^{2}$, which represents the NCC systems, and only one cluster with central entropy lower than $10 \mathrm{keV} \mathrm{cm}^{2}$. 
We observe that from high redshift to low redshift the peak at high entropy becomes less prominent and a larger fraction of clusters get a smaller value of the central entropy, so that in the lower redshift bins the majority of our clusters have a central entropy below $100 \mathrm{keV} \mathrm{cm}^{2}$.

Nevertheless there is an indication of evolution from many NCC systems at high redshift towards mostly relaxed CC clusters at low redshift. Owing to the poor statistics of the sample we are not able to prove this scenario using statistical tests.

\subsection{Evolution with redshift}

We observe, within the central region, an evolution with redshift of the entropy $\left(r<0.1 R_{500}\right)$. It suggests that the entropy profiles are flatter at high redshifts in massive objects, or, alternatively, there is a non-zero central entropy at high redshift. This resembles an evolution in the entropy profiles. However, as we show in Fig. C.2, the fraction of NCC clusters is much higher at high redshifts than at low redshifts. This would imply that at $z>0.77$ the clusters in our dataset are not able to develop a cool core similar to clusters at low redshift, meaning that cool cores are less common in the past, flattening the entropy profiles and creating a non-zero value for the central entropy. Several studies (e.g. Vikhlinin et al. 2007; Santos et al. 2008, 2010; McDonald 2011) that have investigated the evolution of the cool coreness of clusters, support this scenario, in particular on the lower relative abundance of the strongest $\mathrm{CC}$ at high redshift. In other works (e.g. McDonald et al. 2013, 2017), the cuspiness of the gas density is shown to decrease with increasing redshift, as a consequence of a non-evolving core that is embedded in an ambient ICM, which evolves self-similarly.

If we define the CC clusters as those with central entropy that is lower than $100 \mathrm{keV} \mathrm{cm}{ }^{2}$ then from Fig. C.2 we derive that in the low redshift bins $67 \%$ of clusters are $\mathrm{CC}$, which is a percentage that reduces to $50 \%$ and $40 \%$ in the intermediate and high redshift bins, respectively.

This result slightly deviates, but not in a significant way, from what has been presented in previous studies (e.g. Vikhlinin et al. 2007; McDonald et al. 2013). For example, McDonald et al. (2013; see their Fig. 12) obtain that CCs represent $40 \%$ $(10-40 \%)$ of the cluster population at low (high) redshift. Their sample is SZ selected and is, therefore, less biased towards $\mathrm{CC}$ clusters with respect to an X-ray selected sample.

This result is consistent with the hierarchical scenario of the growth of structure, given that at high redshift $(z \sim 1)$ clusters are in the middle of their formation history and cool cores could have easily been destroyed by one of the many merger events, or not even built, if they did not have enough time to relax in the center. Moreover, it is remarkable that even in our sample, which is selected in order to have specific X-ray properties and thus prone to include X-ray bright centrally peaked cool core objects, we observe a relatively larger fraction of NCC at higher redshift.

\subsection{Pressure}

For the same dataset, we study the behaviour of the electronic pressure profile $P(r)=n_{\mathrm{e}}(r) T(r)$.

A generalized NFW profile, as introduced by Nagai et al. (2007) (see Eq. (6)), has been widely used to study the radial rescaled pressure profile. The best-fitting parameters $\left[P_{0}, c_{500}, \gamma, \alpha, \beta\right]=[8.403,1.177,0.3081,1.0510,5.49]$ obtained
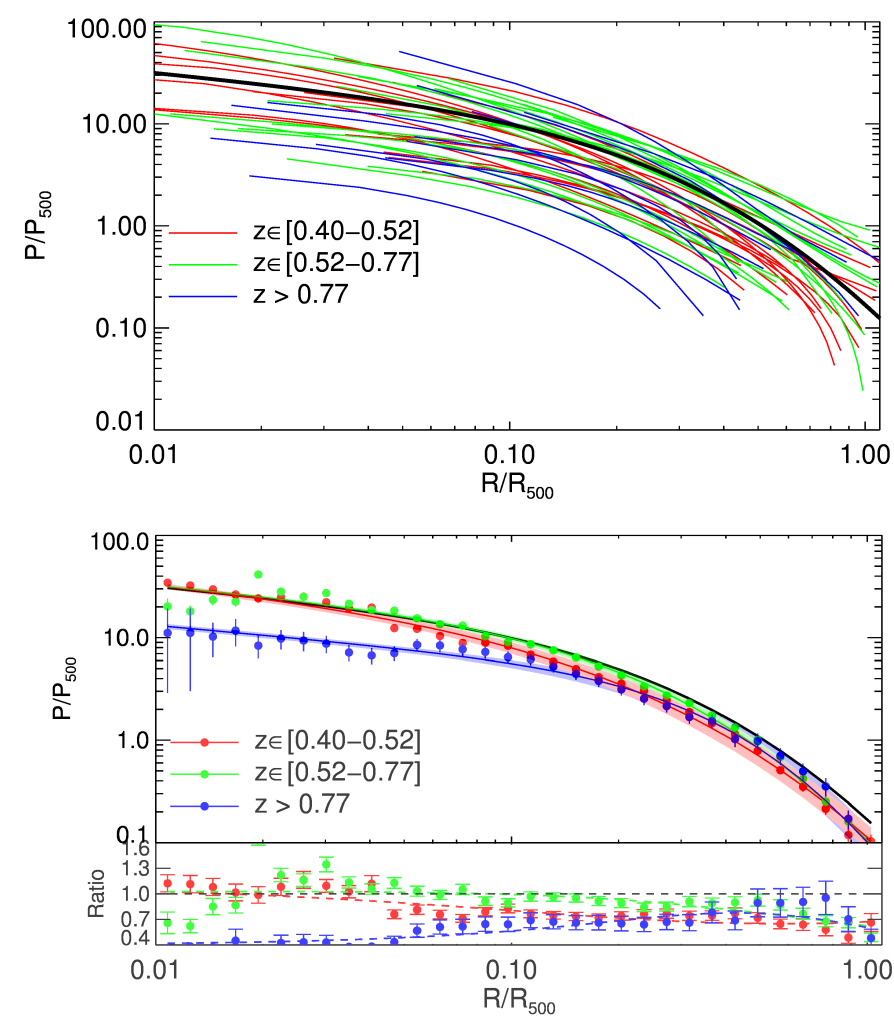

Fig. 9. Top: pressure profiles for all our clusters rescaled using an overdensity of 500, colour coded with respect to the redshift bin to which each curve belongs, compared with the best-fit results in Arnaud et al. (2010; black solid line). Bottom: stacked pressure profiles (in logarithmic space) compared with the best fit of Arnaud et al. (2010); the panel at the bottom shows the ratio with respect to the "universal" pressure profile.

from Arnaud et al. (2010) represent the so-called "universal pressure profile" for galaxy clusters.

Pressure is the quantity less affected by the thermal history of the cluster (Arnaud et al. 2010). Indeed McDonald et al. (2014) found no significant evolution of the pressure profile in the analysis of SPT SZ-selected clusters, just a mild flattening of the profile below $0.1 R_{500}$. Battaglia et al. (2012), however, suggested from the analysis of cosmological hydrodynamical simulations that a significant evolution of the pressure profile should occur beyond $z=0.7$, and only outside $R_{500}$, as consequence of the increasing non-thermal support towards the outskirts of galaxy clusters.

In the functional form shown in Eq. (6), we fix the parameters $\beta$ and $\gamma$ to the fiducial value found in the work of Arnaud et al. (2010). These parameters are degenerate and therefore fixing at least one of the slopes is advised for tighter parameter distributions and a better comparison (Arnaud et al. 2010).

The pressure profiles are plotted in the top panel of Fig. 9, together with the curve of the universal profile, in order to make a comparison. All the profiles show a very similar shape, surrounding the "universal" one from both sides with an apparent discrepancy only in the normalization of the profiles. At each radius the scatter is of about one order of magnitude.

We applied the same procedure described in Sect. 4 and used in the analysis of the entropy profiles. We interpolated over the same radial grid, extrapolated up to $R_{500}$ using the best-fit model, and stacked these pressure profiles. We obtained that the stacked curves in the two low redshift bins are compatible with 
V. Ghirardini et al.: Entropy and pressure profiles in X-ray galaxy clusters at $z>0.4$

Table 2. Best-fitting values, and relative errors for the pressure profiles modelled with the generalized NFW in Eq. (6).

\begin{tabular}{ccccccc}
\hline \hline Subset & $P_{0}$ & $c_{500}$ & $\gamma$ & $\alpha$ & $\beta$ & $\chi_{\text {red }}^{2}$ \\
\hline$z \in[0.4,0.52]$ & $9.1 \pm 0.3$ & $1.08 \pm 0.08$ & 0.308 & $0.87 \pm 0.04$ & 5.49 & 2.1 \\
$z \in[0.52,0.77]$ & $9.2 \pm 0.2$ & $1.48 \pm 0.05$ & 0.308 & $1.11 \pm 0.04$ & 5.49 & 4.6 \\
$z>0.77$ & $3.6 \pm 0.2$ & $1.5 \pm 0.1$ & 0.308 & $1.59 \pm 0.14$ & 5.49 & 0.8 \\
CC & $11.3 \pm 0.3$ & $1.30 \pm 0.07$ & 0.308 & $0.92 \pm 0.03$ & 5.49 & 2.1 \\
NCC & $5.46 \pm 0.14$ & $1.57 \pm 0.05$ & 0.308 & $1.43 \pm 0.06$ & 5.49 & 1.9 \\
& & & & & & \\
Universal & 8.403 & 1.177 & 0.308 & 1.0510 & 5.49 & - \\
\hline
\end{tabular}

Notes. The parameters $\beta$ and $\gamma$ are frozen to their "universal" values to break some degeneracy and facilitate the comparison between the results. The first column indicates the subset of our sample chosen to fit the data. We observe that the results on $c_{500}$ and $\alpha$ of NCC subsample and the result of high redshift subset are compatible within $1 \sigma$. The goodness of the fit is indicated in the last column on the right. In general the value of the parameters are very close to the "universal" results of Arnaud et al. (2010), considering that they are highly degenerate. We point out that the parameter $P_{0}$ for the high redshift bin is significantly smaller than its value for the other bins and the "universal" value.
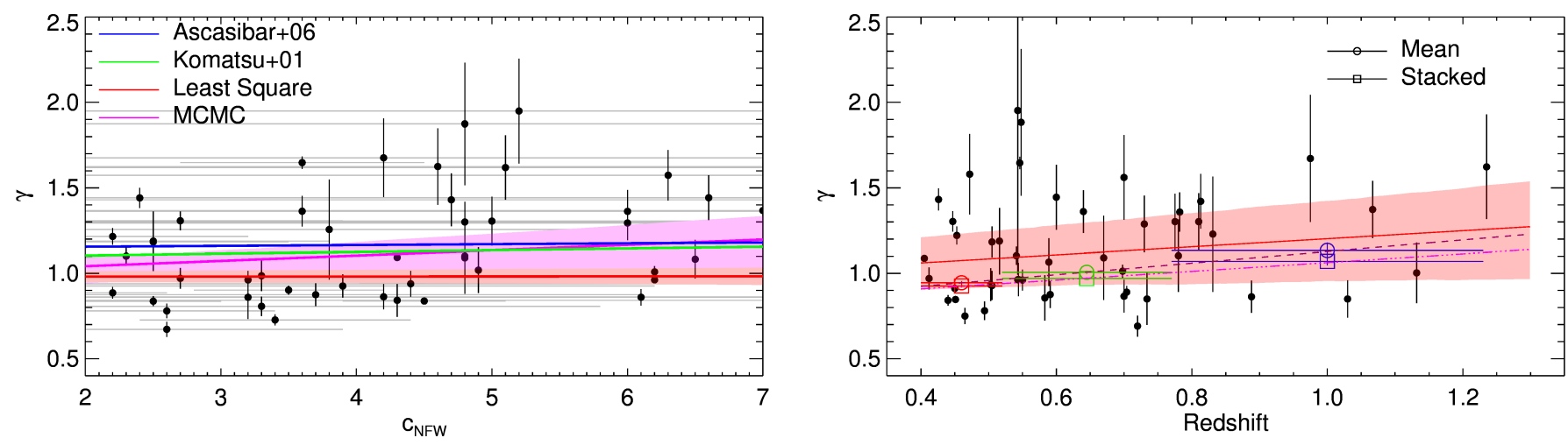

Fig. 10. Left: polytropic index for the objects in our sample vs. concentration: $\gamma$ increases with larger values of $c_{\mathrm{NFW}}$. Black points are the observed data points; the green and blue lines are the results of Komatsu \& Seljak (2001) and Ascasibar et al. (2006), respectively; the red line with pink shaded area is the best fit using classical least squares minimization with $1 \sigma$ confidence region; and the magenta line with lily shaded area is the best fit using an MCMC algorithm. Right: polytropic index for all clusters as a function of redshift. The data are better fitted by a linear relation: the effective polytropic index grows with redshift. Black points indicate the observed data points and the red line with pink shaded area indicates the best fit with $1 \sigma$ dispersion using MCMC algorithm. The empty circles indicate the value of adiabatic index using the mean result for the clusters in each bin; the empty squares indicate the polytropic index of the stacked profiles in each redshift bin; the brown dashed line and dash-dotted magenta line indicate the best linear fit on the "mean" and "stacked" adiabatic index. The parameters describing these lines are listed in Table 3.

the "universal" pressure profile at radii below $0.1 R_{500}$, while above this value the data points are slightly below. Nevertheless the best fit on these stacked data points include the Arnaud et al. (2010) result at all radii within the error bars.

On the other hand, we observe a distinctive flattening below $0.1 R_{500}$ for the high redshift scaled profile for the inner part of these profiles with values that are about $30 \%$ of the "universal" pressure profile. Above this radius the data points are very close to the other redshift bins, meaning slightly below the Arnaud et al. (2010) result.

Pressure is indeed a thermodynamic property that is hardly affected by the thermodynamic history of clusters. The observed flattening at high redshift at low radii is also observed in the work of McDonald et al. (2014) and can be easily explained by the minimal presence of $\mathrm{CC}$ clusters at high redshift.

In Table 2 we show the best fit of Eq. (6), where we have kept $\beta$ and $\gamma$ fixed to the "universal" pressure profile best fit. We obtain a very good fit in all the three redshift bins; only the normalization of this functional in the high redshift bin shows a distinct discrepancy with the results of Arnaud et al. (2010), greater than $5 \sigma$. In fact the bottom panel of Fig. 9 shows that the low redshift bins points are almost on top of the "universal" profile, while the high redshift points are compatible only above $0.1 R_{500}$ and below this threshold the discrepancy grows to be factor 3 at $0.01 R_{500}$. For completeness in the same table we show the fitting performed for the subsets of $\mathrm{CC}$ and NCC, referring to the data in Fig. B.3. We observe that NCC clusters best-fit results resembles the high- $z$ subsample, while $C C$ clusters resemble low- $z$ objects.

\subsection{Polytropic index}

The polytropic index $\gamma$, equal to the ratio of specific heats $C_{P} / C_{V}$ for an ideal gas, is a common proxy when evaluating the physical state and thermal distribution of the gas. It is defined as

$P_{\mathrm{e}}=$ const. $\cdot n_{\mathrm{e}}^{\gamma}$,

with values of $\gamma$ expected to be in the range $[1.1,1.2]$ (Komatsu \& Seljak 2001; Ascasibar et al. 2006; Shi 2016), i.e. between 1 , the value describing an isothermal gas, and 5:3, the value of an isoentropic gas, when the gas is well mixed and the gas entropy per atom is constant.

Studying the evolution of the polytropic index with redshift and its relation with the concentration $c$ of the dark matter distribution can provide a more consistent picture of the processes that regulate the hierarchical structure formation. A correlation between $\gamma$ and $c$ is expected if the radial structure of the ICM and 
Table 3. Value of the polytropic index, dependence on concentration, and evolution with redshift.

\begin{tabular}{ccc}
\hline \hline \multicolumn{3}{c}{ Polytropic index $\gamma$} \\
Mean & Stacked \\
\hline$z \in[0.4,0.52]$ & $0.935 \pm 0.008$ & $0.942 \pm 0.008$ \\
$z \in[0.52,0.77]$ & $0.996 \pm 0.009$ & $0.906 \pm 0.006$ \\
$z>0.77$ & $1.076 \pm 0.031$ & $1.041 \pm 0.018$ \\
\hline \multicolumn{3}{c}{$\gamma=m \cdot c_{\mathrm{NFW}}+q$} \\
Method & $m$ & $q$ \\
\hline Chi-squared & $0.0004 \pm 0.0057$ & $0.98 \pm 0.03$ \\
MCMC & $0.031 \pm 0.020$ & $0.98 \pm 0.09$ \\
Ascasibar+06 & $0.005 \pm 0.002$ & $1.145 \pm 0.007$ \\
Komatsu+01 & 0.01 & 1.085 \\
\hline \multicolumn{3}{c}{$\gamma=m+q$} \\
Method & $m$ & $q$ \\
\hline Chi-squared & $0.05 \pm 0.05$ & $0.94 \pm 0.03$ \\
MCMC & $0.24 \pm 0.22$ & $0.97 \pm 0.14$ \\
Mean & $0.29 \pm 0.05$ & $0.80 \pm 0.030$ \\
Stacked & $0.11 \pm 0.03$ & $0.86 \pm 0.02$ \\
\hline
\end{tabular}

Notes. Top: values and errors on the polytropic index for the three redshift bins considering the mean and stacked values. We observe evolution with a significance greater than $2 \sigma$. Middle: fit of left panel of Fig. 10 using different methods and compared with previous theoretical work. Bottom: fit of right panel of Fig. 10 using different methods.

the host halo depend on the halo mass. Komatsu \& Seljak (2001) require a linear relation between concentration and polytropic index by assuming in this theoretical work that the gas traces the dark matter distribution outside the core. Ascasibar et al. (2006) have shown that $c$ and $\gamma$ conspire to produce the observed scaling relations, matching the self-similar slope at many overdensities.

We estimate an effective polytropic index $\gamma$ by fitting the pressure with a power law as a function of the gas density. As a first step, $\gamma$ is calculated for each single cluster. Then, we evaluate the weighted mean in each bin. We also calculate $\gamma$ for the stacked profiles.

We look for correlations between the polytropic index and dark matter concentration as recovered from the best fit with a NFW model in Amodeo et al. (2016). Ascasibar et al. (2006) and Komatsu \& Seljak (2001) have shown that between concentration and polytropic index there is a linear relation with slope of 0.005 and 0.01 , respectively. Using the Markov chain Monte Carlo (MCMC) code emcee (Foreman-Mackey et al. 2013), we obtain a slope that is much stepper than the theoretical predictions, although with a relative uncertainty of about $70 \%$ (see best-fit values in Table 3), which makes it compatible with previous results (Komatsu \& Seljak 2001; Ascasibar et al. 2006) within $1 \sigma$, and even compatible with 0 at $2 \sigma$. Moreover, the intercept we get is much smaller than what has been previously calculated.

In the right panel of Fig. 10, we show the polytropic index as a function of redshift. We measure a positive evolution with redshift, with larger values of $\gamma$ (by more than $2 \sigma$ ) at higher redshift (see Table 3).

\section{Conclusions}

From the sample described in Amodeo et al. (2016), which contains one of the largest collections of clusters at $z>0.8$ that are homogeneously analysed in their X-ray spectral properties, we have extracted the entropy and pressure profiles of 47 clusters observed with Chandra in a redshift range from 0.4 to 1.24.

We observe higher values of the gas entropy in the central region at higher redshift, which cannot be explained as an effect owing to spatial resolution. A plausible explanation of this result is the fact that at high redshift we observe a lack of cool core clusters with respect to the low redshift sample.

Moreover at intermediate radii, between $0.1 R_{500}$ and $0.7 R_{500}$, the self-similarity is recovered when we use entropy dependence on both the redshift and gas fraction and the scatter between the profiles is reduced by a factor $\sim 3$. The best fit of the stacked profiles is very similar to the Voit et al. (2005) prediction from non-radiative simulations. We also show that the pressure profiles flatten at high redshift at radii below $0.1 R_{500}$ with lower values by about $50 \%$ than those observed at $z \lesssim 0.5$.

Overall, these results agrees with a scenario in which galaxy clusters are the last gravitationally bound structures to form according to the hierarchical evolution. They start forming at $z \approx 3$, and at $z \sim 1-1.5$ they are still in the middle of their formation. At this epoch, cool cores could either be destroyed by merger events or have not formed yet, thereby reducing their relative number at earlier epoch. Moreover, the merging processes ongoing at high redshift would imply that objects at $z \sim 1$ are mostly unrelaxed, with a flatter entropy profile, which produces a clear excess in the inner parts and a deficit in the outskirts. As we show in Figs. 5 and 6, high redshift clusters indeed have a rather flat stacked entropy profile, supporting the evidence that the floating and sinking of the gas entropy has not been completed yet. The thermodynamical disturbed condition of the high redshift systems is further supported by the observed flattening of thermal pressure in the inner part of the stacked pressure profile (see Fig. 9).

Moreover, we measure a slightly significant evolution of the effective polytropic index of the ICM, we measure it by estimating $\mathrm{d} \log P_{\mathrm{e}} / \mathrm{d} \log n_{\mathrm{e}}$, using the dark matter concentration and redshift with an $87 \%$ significance $(1.5 \sigma)$ for concentration and $73 \%(1.1 \sigma)$ for redshift, indicating that the gas possesses a slightly larger polytropic index in systems that have a more concentrated mass distribution at higher redshift. This result supports the observational evidence that at high redshift we recover more isentropic (i.e. more flat) entropy profiles.

\section{References}

Allen, S. W., Schmidt, R. W., Ebeling, H., Fabian, A. C., \& van Speybroeck, L. 2004, MNRAS, 353, 457

Amodeo, S., Ettori, S., Capasso, R., \& Sereno, M. 2016, A\&A, 590, A126 Arnaud, M., Pratt, G. W., Piffaretti, R., et al. 2010, A\&A, 517, A92, 11 Ascasibar, Y., Sevilla, R., Yepes, G., Müller, V., \& Gottlöber, S. 2006, MNRAS, 371,193

Bartalucci, I., Arnaud, M., Pratt, G. W., et al. 2017, A\&A, 598, A61 Battaglia, N., Bond, J. R., Pfrommer, C., \& Sievers, J. L. 2012, ApJ, 758, 75

Binney, J., \& Tremaine, S. 1987, Galactic dynamics (Princeton, NJ; Princeton University Press)

Böhringer, H., Voges, W., Huchra, J. P., et al. 2000, ApJS, 129, 435 Bonamente, M., Joy, M. K., LaRoque, S. J., et al. 2006, ApJ, 647, 25 Borgani, S., Governato, F., Wadsley, J., et al. 2001, ApJ, 559, L71 Bryan, G. L. 2000, ApJ, 544, L1

Cassano, R., Ettori, S., Giacintucci, S., et al. 2010, ApJ, 721, L82 Cavagnolo, K. W., Donahue, M., Voit, G. M., \& Sun, M. 2009, ApJS, 182, 12 Coble, K., Bonamente, M., Carlstrom, J. E., et al. 2007, AJ, 134, 897 De Propris, R., Stanford, S. A., Eisenhardt, P. R., Holden, B. P., \& Rosati, P. 2007, AJ, 133, 2209

Donahue, M., Voit, G. M., O’Dea, C. P., Baum, S. A., \& Sparks, W. B. 2005 , ApJ, 630, L13

Ebeling, H., Jones, L. R., Fairley, B. W., et al. 2001, ApJ, 548, L23

Ebeling, H., Barrett, E., Donovan, D., et al. 2007, ApJ, 661, L33

Edge, A. C., Ebeling, H., Bremer, M., et al. 2003, MNRAS, 339, 913 
Ellis, S. C., \& Jones, L. R. 2004, MNRAS, 348, 165

Ettori, S., Donnarumma, A., Pointecouteau, E., et al. 2013, Space Sci. Rev., 177, 119

Fassbender, R., Böhringer, H., Santos, J. S., et al. 2011, A\&A, 527, A78

Foley, R. J., Andersson, K., Bazin, G., et al. 2011, ApJ, 731, 86

Foreman-Mackey, D., Hogg, D. W., Lang, D., \& Goodman, J. 2013, PASP, 125, 306

Gioia, I. M., \& Luppino, G. A. 1994, ApJS, 94, 583

Gioia, I. M., Braito, V., Branchesi, M., et al. 2004, A\&A, 419, 517

Gonzalez, A. H., Zaritsky, D., Dalcanton, J. J., \& Nelson, A. 2001, ApJS, 137, 117

Gralla, M. B., Sharon, K., Gladders, M. D., et al. 2011, ApJ, 737, 74

Henry, J. P., Gioia, I. M., Mullis, C. R., et al. 1997, AJ, 114, 1293

Hicks, A. K., Ellingson, E., Bautz, M., et al. 2008, ApJ, 680, 1022

High, F. W., Stalder, B., Song, J., et al. 2010, ApJ, 723, 1736

Holden, B. P., Stanford, S. A., Squires, G. K., et al. 2002, AJ, 124, 33

Komatsu, E., \& Seljak, U. 2001, MNRAS, 327, 1353

Kotov, O., \& Vikhlinin, A. 2006, ApJ, 641, 752

Mantz, A. B., Allen, S. W., Morris, R. G., et al. 2014, MNRAS, 440, 2077

Markwardt, C. B. 2009, Astronomical Data Analysis Software and Systems XVIII, ASP Conf. Ser., 411, 251

McCarthy, I. G., Schaye, J., Bird, S., \& Le Brun, A. M. C. 2017, MNRAS, 465, 2936

McDonald, M. 2011, ApJ, 742, L35

McDonald, M., Benson, B. A., Vikhlinin, A., et al. 2013, ApJ, 774, 23

McDonald, M., Benson, B. A., Vikhlinin, A., et al. 2014, ApJ, 794, 67

McDonald, M., Allen, S. W., Bayliss, M., et al. 2017, ApJ, 843, 28

Morandi, A., \& Ettori, S. 2007, MNRAS, 380, 1521

Morandi, A., \& Cui, W. 2014, MNRAS, 437, 1909

Mullis, C. R., McNamara, B. R., Quintana, H., et al. 2003, ApJ, 594, 154

Muñoz-Cuartas, J. C., Macciò, A. V., Gottlöber, S., \& Dutton, A. A. 2011, MNRAS, 411, 584

Nagai, D., Kravtsov, A. V., \& Vikhlinin, A. 2007, ApJ, 668, 1
Navarro, J. F., Frenk, C. S., \& White, S. D. M. 1997, ApJ, 490, 493

Panagoulia, E. K., Fabian, A. C., \& Sanders, J. S. 2014, MNRAS, 438, 2341 Perlman, E. S., Horner, D. J., Jones, L. R., et al. 2002, ApJS, 140, 265 Peterson, J. R., \& Fabian, A. C. 2006, Phys. Rep., 427, 1

Planck Collaboration XIII. 2016, A\&A, 594, A13

Ponman, T. J., Cannon, D. B., \& Navarro, J. F. 1999, Nature, 397, 135 Pratt, G. W., Croston, J. H., Arnaud, M., Bohringer, H. 2009, A\&A, 498, 361 Pratt, G. W., Arnaud, M., Piffaretti, R., et al. 2010, A\&A, 511, A85 Romer, A. K., Nichol, R. C., Holden, B. P., et al. 2000, ApJS, 126, 209 Rosati, P., Tozzi, P., Ettori, S., et al. 2004, AJ, 127, 230

Santos, J. S., Rosati, P., Tozzi, P., et al. 2008, A\&A, 483, 35

Santos, J. S., Tozzi, P., Rosati, P., Bohringer, H. 2010, A\&A, 521, A64

Shi, X. 2016, MNRAS, 461, 1804

Schindler, S., Guzzo, L., Ebeling, H., et al. 1995, A\&A, 299, L9

Schwarz G. 1978, Ann. Statist., 5, 461

Sereno, M., \& Ettori, S. 2015, MNRAS, 450, 3675

Song, J., Zenteno, A., Stalder, B., et al. 2012, ApJ, 761, 22

Stocke, J. T., Morris, S. L., Gioia, I. M., et al. 1991, ApJS, 76, 813

Sunyaev, R. A., \& Zeldovich, I. B. 1980, ARA\&A, 18, 537

Takey, A., Schwope, A., \& Lamer, G. 2011, A\&A, 534, A120

Tozzi, P., \& Norman, C. 2001, ApJ, 546, 63

Tozzi, P., Scharf, C., \& Norman, C. 2000, ApJ, 542, 106

Vanderlinde, K., Crawford, T. M., de Haan, T., et al. 2010, ApJ, 722, 1180

Vikhlinin, A., McNamara, B. R., Forman, W., et al. 1998, ApJ, 502, 558

Vikhlinin, A., Burenin, R., Forman, W. R., et al. 2007, in Heating versus Cooling in Galaxies and Clusters of Galaxies (Berlin Heiderlberg: Springer-Verlag), ESO Astrophys. Symp., 48

Voit, G. M. 2005, Rev. Mod. Phys., 77, 207

Voit, G. M., Kay, S. T., Bryan G. L. 2005, MNRAS, 364, 909

Wang, Q. D., \& Walker, S. 2014, MNRAS, 439, 1796

Wen, Z. L., Han, J. L., \& Liu, F. S. 2009, ApJS, 183, 197

Zhuravleva, I., Churazov, E., Schekochihin, A. A., et al. 2014, Nature, 515, 85 


\section{Appendix A: Additional information}

Table A.1. Addition to Table 2 in Amodeo et al. (2016): information on the sizes and gas content of these clusters.

\begin{tabular}{|c|c|c|c|c|}
\hline Object & $\begin{array}{l}z \\
-\end{array}$ & $\begin{array}{l}R_{500} \\
{[\mathrm{kpc}]}\end{array}$ & $\underset{\text { [fraction] }}{f_{\text {gas } 500}}$ & Reference for redshift \\
\hline MACS0159.8-084 & 0.405 & $1379 \pm 111$ & $0.11 \pm 0.02$ & Kotov \& Vikhlinin (2006) \\
\hline MACSJ2228.5+20 & 0.412 & $1418 \pm 156$ & $0.13 \pm 0.03$ & Böhringer et al. (2000) \\
\hline MS1621.5+2640 & 0.426 & $1298 \pm 127$ & $0.1 \pm 0.02$ & Stocke et al. (1991) \\
\hline MACSJ1206.2-08 & 0.44 & $1874 \pm 128$ & $0.08 \pm 0.01$ & Borgani et al. (2001) \\
\hline MACS-J2243.3-0 & 0.447 & $1335 \pm 137$ & $0.16 \pm 0.03$ & Coble et al. (2007) \\
\hline MACS0329.7-021 & 0.45 & $1264 \pm 113$ & $0.12 \pm 0.04$ & Allen et al. (2004) \\
\hline RXJ1347.5-1145 & 0.451 & $1756 \pm 134$ & $0.1 \pm 0.02$ & Schindler et al. (1995) \\
\hline $\mathrm{V} 1701+6414$ & 0.453 & $707 \pm 48$ & $0.26 \pm 0.04$ & Wang \& Walker (2014) \\
\hline MACS1621.6+381 & 0.465 & $1349 \pm 242$ & $0.09 \pm 0.04$ & Edge et al. (2003) \\
\hline CL0522-3624 & 0.472 & $754 \pm 271$ & $0.11 \pm 0.11$ & Mullis et al. (2003) \\
\hline MACS1311.0-031 & 0.494 & $1420 \pm 241$ & $0.06 \pm 0.02$ & Allen et al. (2004) \\
\hline MACS-J2214.9-1 & 0.503 & $1275 \pm 253$ & $0.14 \pm 0.06$ & Bonamente et al. (2006) \\
\hline MACS911.2+1746 & 0.505 & $1338 \pm 140$ & $0.1 \pm 0.07$ & Ebeling et al. (2007) \\
\hline MACSJ0257.1-23 & 0.505 & $1293 \pm 260$ & $0.12 \pm 0.05$ & Ebeling et al. (2007) \\
\hline V1525+0958 & 0.516 & $1259 \pm 191$ & $0.05 \pm 0.01$ & Mullis et al. (2003) \\
\hline MS0015.9+1609 & 0.541 & $1381 \pm 128$ & $0.13 \pm 0.02$ & Stocke et al. (1991) \\
\hline CL0848.6+4453 & 0.543 & $701 \pm 394$ & $0.13 \pm 0.2$ & Takey et al. (2011) \\
\hline MACS1423.8+240 & 0.543 & $1041 \pm 16$ & $0.13 \pm 0.01$ & Ebeling et al. (2007) \\
\hline MACSJ1149.5+22 & 0.544 & $1187 \pm 141$ & $0.19 \pm 0.04$ & Ebeling et al. (2007) \\
\hline MACSJ0717.5+3745 & 0.546 & $1409 \pm 59$ & $0.17 \pm 0.02$ & Ebeling et al. (2007) \\
\hline CL1117+1744 & 0.548 & $568 \pm 106$ & $0.2 \pm 0.09$ & Wen et al. (2009) \\
\hline MS0451.6-0305 & 0.55 & $1699 \pm 229$ & $0.07 \pm 0.03$ & Gioia \& Luppino (1994) \\
\hline MS2053.7-0449 & 0.583 & $1239 \pm 367$ & $0.04 \pm 0.02$ & Stocke et al. (1991) \\
\hline MACS-J2129.4-0 & 0.589 & $1155 \pm 228$ & $0.15 \pm 0.07$ & Ebeling et al. (2007) \\
\hline MACS-J0647.7+7 & 0.591 & $1762 \pm 333$ & $0.06 \pm 0.02$ & Ebeling et al. (2007) \\
\hline CL1120+4318 & 0.6 & $894 \pm 208$ & $0.16 \pm 0.08$ & Romer et al. (2000) \\
\hline CLJ0542.8-4100 & 0.64 & $801 \pm 68$ & $0.13 \pm 0.03$ & De Propris et al. (2007) \\
\hline LCDCS954 & 0.67 & $567 \pm 98$ & $0.16 \pm 0.07$ & Gonzalez et al. (2001) \\
\hline MACS0744.9+392 & 0.698 & $994 \pm 93$ & $0.17 \pm 0.04$ & Ebeling et al. (2007) \\
\hline SPT-CL0001-5748 & 0.7 & $902 \pm 261$ & $0.12 \pm 0.08$ & Vikhlinin et al. (1998) \\
\hline $\mathrm{V} 1221+4918$ & 0.7 & $738 \pm 127$ & $0.15 \pm 0.07$ & Mantz et al. (2014) \\
\hline RCS2327.4-0204 & 0.704 & $1547 \pm 112$ & $0.08 \pm 0.01$ & Gralla et al. (2011) \\
\hline SPT-CLJ2043-5035 & 0.72 & $1348 \pm 255$ & $0.07 \pm 0.02$ & Song et al. (2012) \\
\hline ClJ1113.1-2615 & 0.73 & $747 \pm 178$ & $0.06 \pm 0.04$ & Perlman et al. (2002) \\
\hline CLJ2302.8+0844 & 0.734 & $1042 \pm 207$ & $0.06 \pm 0.02$ & Perlman et al. (2002) \\
\hline SPT-CL2337-5942 & 0.775 & $1590 \pm 414$ & $0.04 \pm 0.02$ & Vanderlinde et al. (2010) \\
\hline RCS2318+0034 & 0.78 & $1681 \pm 484$ & $0.02 \pm 0.01$ & Hicks et al. (2008) \\
\hline MS1137.5+6625 & 0.782 & $994 \pm 203$ & $0.06 \pm 0.03$ & Gioia \& Luppino (1994) \\
\hline RXJ1350.0+6007 & 0.81 & $577 \pm 71$ & $0.17 \pm 0.05$ & Holden et al. (2002) \\
\hline RXJ1716.9+6708 & 0.813 & $646 \pm 89$ & $0.15 \pm 0.05$ & Henry et al. (1997) \\
\hline EMSS1054.5-0321 & 0.831 & $1308 \pm 227$ & $0.06 \pm 0.02$ & Gioia et al. (2004) \\
\hline CLJ1226.9+3332 & 0.888 & $1752 \pm 409$ & $0.04 \pm 0.02$ & Ebeling et al. (2001) \\
\hline XMMUJ1230+1339 & 0.975 & $792 \pm 309$ & $0.08 \pm 0.07$ & Fassbender et al. (2011) \\
\hline $\mathrm{J} 1415.1+3612$ & 1.03 & $772 \pm 259$ & $0.08 \pm 0.05$ & Ellis \& Jones (2004) \\
\hline SPT-CL0547-5345 & 1.067 & $779 \pm 188$ & $0.14 \pm 0.08$ & High et al. (2010) \\
\hline SPT-CLJ2106-584 & 1.132 & $963 \pm 254$ & $0.13 \pm 0.06$ & Foley et al. (2011) \\
\hline RDCS1252-29 & 1.235 & $533 \pm 124$ & $0.13 \pm 0.08$ & Rosati et al. (2004) \\
\hline
\end{tabular}

\section{Appendix B: CC versus NCC}

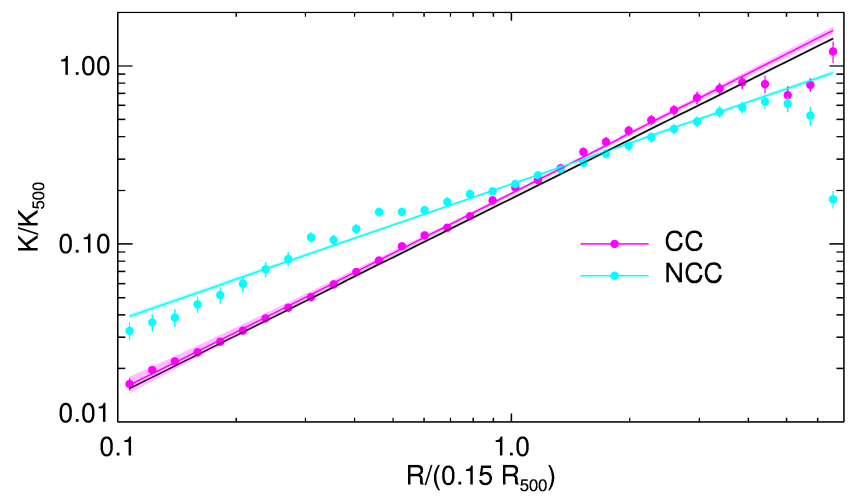

Fig. B.1. Staked entropy profiles for the subsets of CC and NCC based on Cassano et al. (2010) criteria. We observe that CC profile is almost on top of the Voit et al. (2005) prediction.

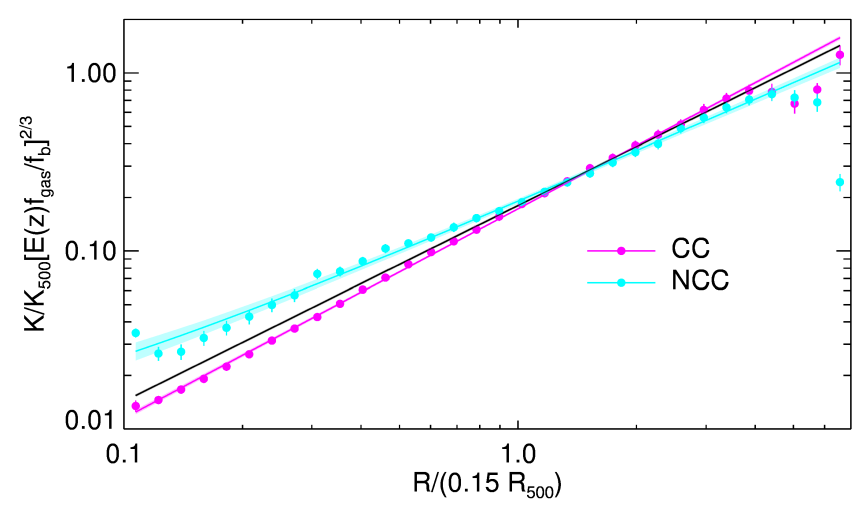

Fig. B.2. Stacked entropy profiles corrected using the gas fraction for the subsets of CC and NCC. The CC entropy profile still lies very close to the prediction, while the NCC profile has gotten closer but still flatter than the prediction.

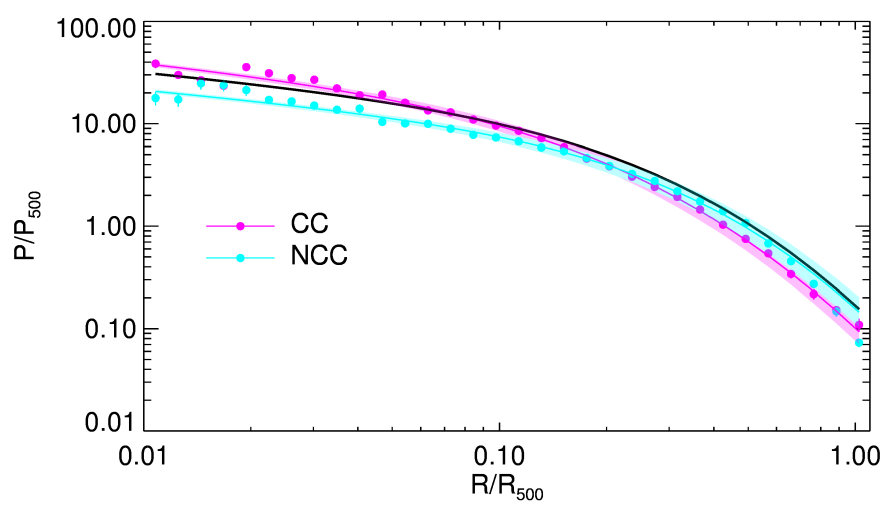

Fig. B.3. Pressure profiles for the subsets of CC and NCC. These profiles are very similar to those in Fig. 9, however the NCC profiles within $0.1 R_{500}$ are about a factor of 2 higher than the high redshift one. 


\section{Appendix C: Plots}
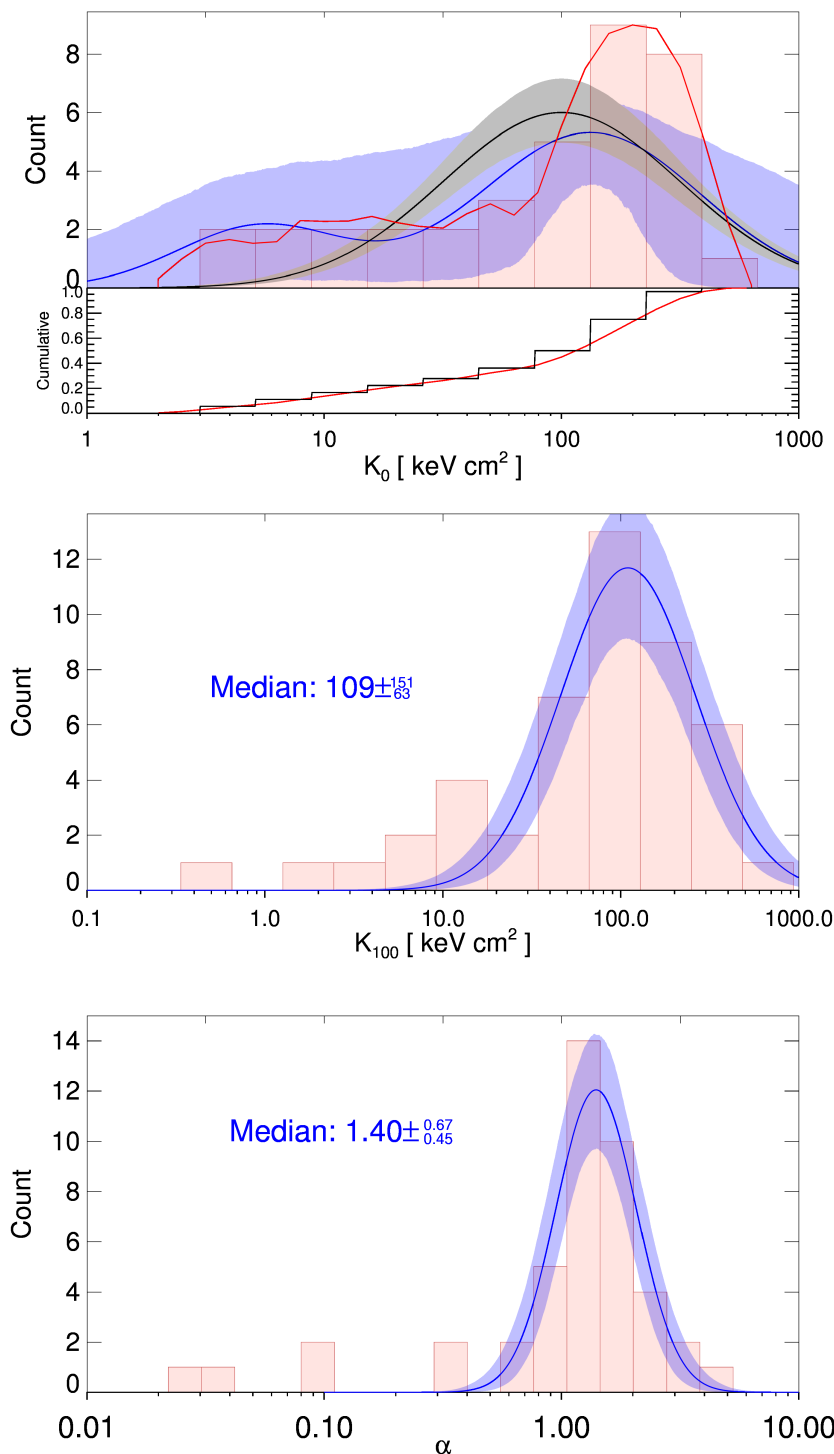

Fig. C.1. Top: distribution of the parameter $K_{0}$. The red line represents the kernel density plot with a smoothing width of 0.1 . The black and blue line represens the best fit obtained using one and two lognormal distributions, respectively. The shaded grey and blue regions enclose the $68.3 \%$ probability region around the best fit due to parameter uncertainties. Middle and bottom: distribution of the parameters $K_{100}$ and $\alpha$, respectively. The blue line with shaded area represents the best fit with a single lognormal distribution and the $1 \sigma$ probability regions around it. The median value of the fit is shown directly on the graph.
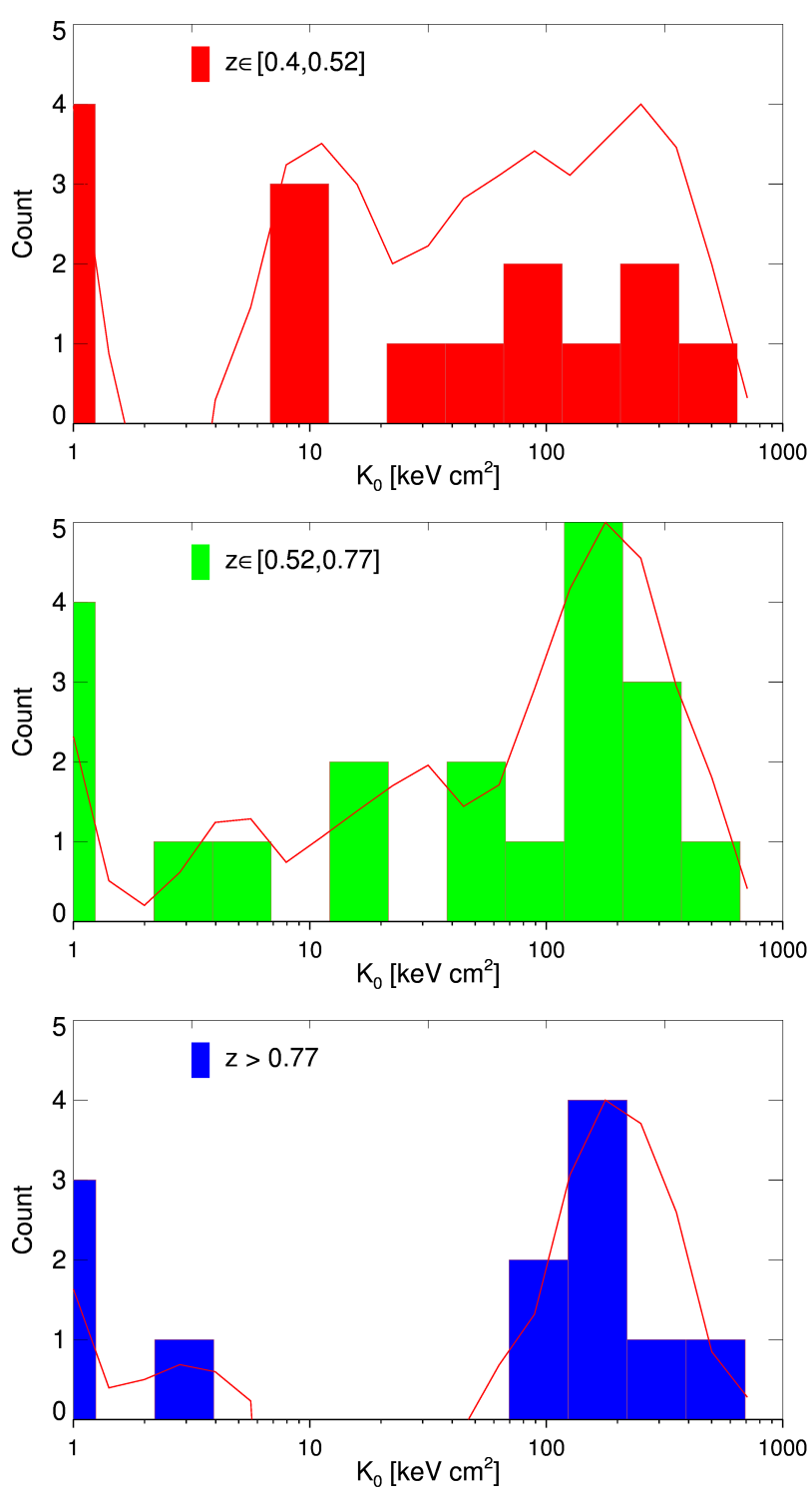

Fig. C.2. Evolution of the central entropy distribution with redshift. We can clearly see an evolution with evidence of bimodality at high redshift. The red lines represents the kernel density estimation of the distributions. The clusters that are best fitted by $K_{0}=0$ are added as if they have the value of $1 \mathrm{kev} \mathrm{cm}^{2}$. 

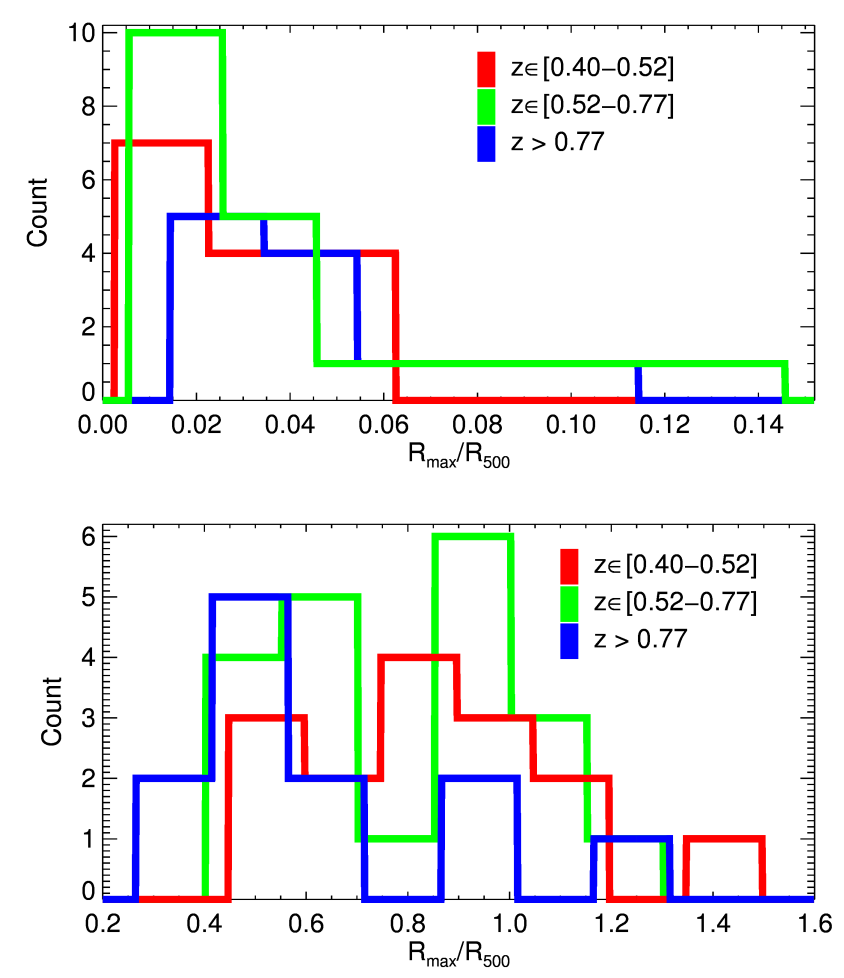

Fig. C.3. Distribution of the rescaled innermost (top) and outermost (bottom) radial spatial bin colour coded with redshift.

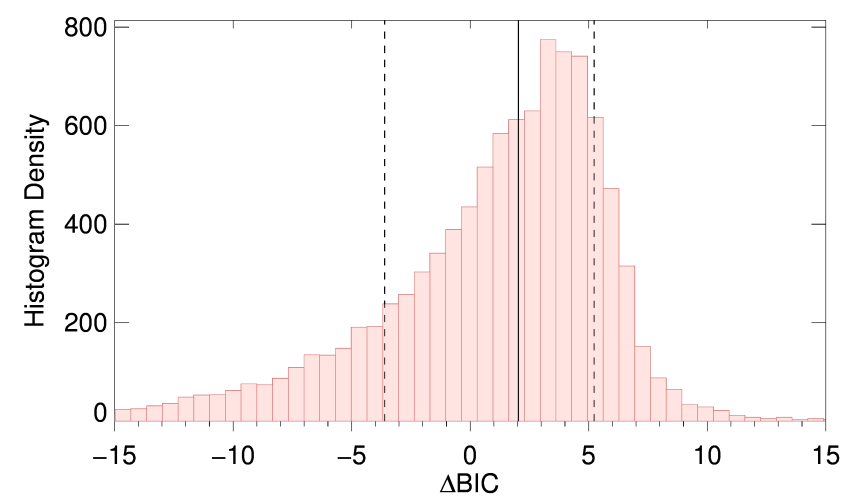

Fig. C.4. Distribution of the bootstraps results. The black solid line represents the position of the median, while the dashed lines represent the region comprehending $68 \%$ of the distribution. The $1 \sigma$ region is compatible with $\triangle \mathrm{BIC}=0$.
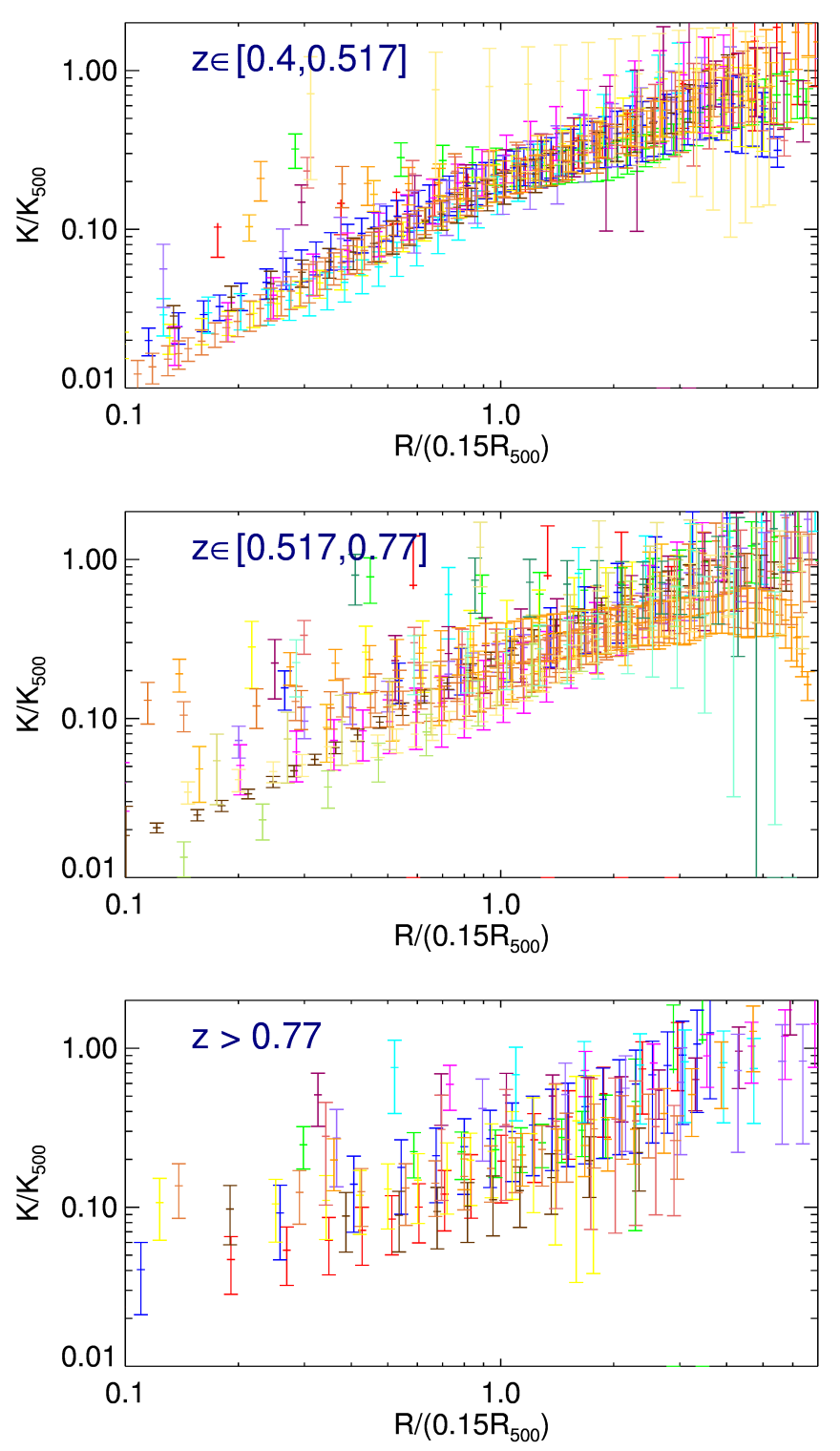

Fig. C.5. Rescaled entropy profiles of clusters in three redshift bins. Each colour represents data from a single cluster. 